\title{
On Computing the Mordukhovich Subdifferential Using Directed Sets in Two Dimensions
}

Robert Baier, Elza Farkhi, and Vera Roshchina

Robert Baier

Chair of Applied Mathematics, University of Bayreuth, D-95440 Bayreuth, Germany, e-mail: robert.baier@uni-bayreuth.de Elza Farkhi

School of Mathematical Sciences, Sackler Faculty of Exact Sciences, Tel Aviv University, 69978 Tel Aviv, Israel, e-mail: elza@post.tau.ac.il

Vera Roshchina

Centro de Investigação em Matemática e Aplicações, Universidade de Évora, Colégio Luís Verney, Rua Romão Ramalho, 59, $7000-671$ Évora, Portugal, e-mail: vera.roshchina@gmail.com 

Dedicated to Boris Mordukhovich on his sixtieth birthday. 
Abstract The Mordukhovich subdifferential, being highly important in variational and non-smooth analysis and optimization, often happens to be hard to calculate. We propose a method for computing the Mordukhovich subdifferential of differences of sublinear (DS) functions via the directed subdifferential of differences of convex (DC) functions. We restrict ourselves to the two-dimensional case mainly for simplicity of the proofs and for the visualizations.

The equivalence of the Mordukhovich symmetric subdifferential (the union of the corresponding subdifferential and superdifferential) to the Rubinov subdifferential (the visualization of the directed subdifferential) is established for DS functions in two dimensions. The Mordukhovich subdifferential and superdifferential are identified as parts of the Rubinov subdifferential. In addition, it is possible to construct the directed subdifferential in a way similar to the Mordukhovich one by considering outer limits of Fréchet subdifferentials. The results are extended to the case of DC functions. Examples illustrating the obtained results are presented.

2010 Mathematics Subject Classification. Primary 49J52; Secondary 26B25, 49J50, 90C26

Key words: non-convex subdifferentials and superdifferentials (basic subdifferential, Rubinov subdifferential), Fréchet subdifferential, difference of convex (DC) functions, differences of sets

\section{Introduction}

The Mordukhovich subdifferential is a highly important notion in variational analysis, closely related to optimality conditions, metric regularity, Lipschitzness and other fundamental concepts of modern optimization theory (see $[23,24])$. This subdifferential is a closed subset of the Clarke subdifferential (see e.g. [25, Theorem 9.2]), and may be non-convex for non-convex functions, thus achieving sharper optimality conditions. In contrast to the Fréchet subdifferential (cf. [18, Example 1.1]), the Mordukhovich subdifferential of a locally Lipschitz function is always nonempty (see e.g. [22, (2.17)]).

Along with these essential advantages, there comes a substantial drawback: the Mordukhovich subdifferential is difficult to calculate even for relatively simple examples, as such computation normally involves finding the Painlevé-Kuratowski outer limit (see Section 2). For most known subdifferentials, the sum rule only has the form of an inclusion - the subdifferential of a sum is a subset of the sum of the subdifferentials [23, Theorem 3.36]. This rule applied in calculations only provides a superset of the subdifferential of the sum.

We propose a method for computing the Mordukhovich subdifferential of differences of sublinear (DS) functions, which are positively homogeneous DC (difference of convex) functions, applying directed sets [2] and the directed subdifferential of DC functions [4]. The DC functions represent a large family of functions. They are dense in the space of continuous functions [16] and constitute an important subclass of the quasidifferentiable functions [10]. Various aspects of calculus and optimality conditions for this class of functions are discussed e.g. in $[1,8,10,11,12,14,20]$.

The class of positively homogeneous DC functions is important enough since it contains differences of support functions and directional derivatives of DC functions. Many interesting examples of non-convex DC functions in the literature are in this class (see e.g. [4]). All results in Section 3 obtained first for DS functions can be formulated as a corollary for the directional derivative of DC functions.

The main advantage of directed subdifferentials based on directed sets is the sum rule: the directed subdifferential of a sum is equal to the sum of the directed subdifferentials [4, Proposition 4.2]. This rule applied for directed subdifferentials provides the exact result.

We restrict ourselves to the two-dimensional case mainly for simplicity of the proofs and for the visualizations. Furthermore, the visualization of the directed subdifferential is essentially more complicated in dimensions higher than two, since lower dimensional mixed-type parts missing in the two-dimensional case would emerge in higher dimensions.

In this paper, the equivalence of the Mordukhovich symmetric subdifferential, the union of the corresponding subdifferential and superdifferential, to the Rubinov subdifferential (the visualization of the directed subdifferential), is established in Theorem 3.14 for the special class of DS functions in two dimensions.

While the Mordukhovich subdifferential is based on the corresponding normal cone and can be calculated by outer limits of the Fréchet subdifferential, the directed subdifferential for DC functions is essentially based on the 
subtraction of convex subdifferentials embedded in the Banach space of directed sets. Although these two concepts differ substantially, there are many interesting links between them.

In Theorem 3.13 we prove that certain parts of the Rubinov subdifferential comprise the Mordukhovich subdifferential. The remaining parts coincide with the Mordukhovich superdifferential (see Theorem 3.14). Furthermore, Theorem 3.11 links outer limits of the Fréchet subdifferential to the directed subdifferential. The assumption on positive homogeneity of the DC functions is dropped in Theorems 3.16 and 3.17 yielding the connection of the Rubinov subdifferential to the Mordukhovich symmetric subdifferential of the directional derivative for the broader class of DC functions.

The paper is organized as follows. In the next section we recall necessary definitions, notation and results on Fréchet subdifferential. In Sect. 3 the relation between the Mordukhovich and the directed subdifferential is discussed. We illustrate our results with several examples in Sect. 4. In the last section we sketch directions for future research.

\section{Preliminaries}

Recall that $f: \mathbb{R}^{n} \rightarrow \mathbb{R}$ is called positively homogeneous, if $f(\lambda x)=\lambda f(x)$ for all $x \in \mathbb{R}^{n}$ and $\lambda>0$. Clearly, $f(0)=0$ for positively homogeneous functions. A function is sublinear if it is convex and positively homogeneous. Recall that support functions of compact sets are sublinear. We denote by $\mathscr{S}_{n-1}$ the unit sphere in $\mathbb{R}^{n}$, and by $\operatorname{cl}(A), \operatorname{co}(A)$ the closure and the convex hull of the set $A$ respectively. The following operations on sets $A, B \subset \mathbb{R}^{n}$ are well-known:

$$
\begin{aligned}
A+B & :=\{a+b \mid a \in A, b \in B\} & & \text { (Minkowski addition), } \\
\ominus A & :=\{-a \mid a \in A\} & & \text { (the pointwise negative of the set } A \text { ) }
\end{aligned}
$$

The last operation is used in the definition of the Mordukhovich superdifferential and in the negative part of the visualization of the directed subdifferential.

For the sets $A, B \subset \mathbb{R}^{n}$ the operation

$$
A \stackrel{*}{*}=\left\{x \in \mathbb{R}^{n} \mid x+B \subset A\right\}=\bigcap_{b \in B}(A-b)
$$

is called the geometric difference of the sets $A$ and $B$. This difference is introduced by Hadwiger in [13] as well as in [28] and is also called Minkowski-Pontryagin difference.

Let $C \subset \mathbb{R}^{n}$ be nonempty, convex, compact and $l \in \mathbb{R}^{n}$. Then, the support function and respectively the supporting face of $C$ in direction $l$ are defined by

$$
\begin{aligned}
\delta^{*}(l, C) & =\max _{c \in C}\langle l, c\rangle, \\
Y(l, C) & =\left\{y \in C \mid\langle l, y\rangle=\delta^{*}(l, C)\right\}=\arg \max _{c \in C}\langle l, c\rangle .
\end{aligned}
$$

Note that for $l=0, Y(l, C)=C$. By $y(l, C)$ we denote any point of the set $Y(l, C)$, and if the latter is a singleton (i.e., there is a unique supporting point), then $Y(l, C)=\{y(l, C)\}$.

The supporting face $Y(l, C)$ equals the subdifferential of the support function of $C$ at $l$ [29, Corollary 23.5.3].

We denote by Limsup the Painlevé-Kuratowski outer limit and by Liminf the inner limit of sets (see [30, Chap. 4]). Intuitively, the outer limit of a sequence of sets consists of the limiting points of all converging subsequences of points from these sets. In contrast, the inner limit consists of limiting points of all sequences constructed from points taken from almost every set in a way that only a finite number of sets can be missed out. For a more rigorous definition (see [30, Sect. 4.A]), first consider the set $\mathscr{N}_{\infty}^{\sharp}$ of all infinite subsequences in the set of natural numbers $\mathscr{N}_{\infty}^{\sharp}:=\{N \subset \mathbb{N} \mid N$ infinite $\}$, and the set $\mathscr{N}_{\infty}$ of all the sequences of natural numbers which include all numbers beyond a certain value, i.e. $\mathscr{N}_{\infty}:=\{N \subset \mathbb{N} \mid N \backslash N$ finite $\}$. Given a sequence $\left\{C_{k}\right\}$ of sets in $\mathbb{R}^{n}$, we set

$$
\begin{gathered}
\underset{k \rightarrow \infty}{\operatorname{Limsup}} C_{k}=\left\{x \in \mathbb{R}^{n} \mid \exists N \in \mathscr{N}_{\infty}^{\sharp}, \exists x_{k} \in C_{k}(k \in N) \text { with } x_{k} \rightarrow x\right\}, \\
\underset{k \rightarrow \infty}{\operatorname{Liminf}} C_{k}=\left\{x \in \mathbb{R}^{n} \mid \exists N \in \mathscr{N}_{\infty}, \exists x_{k} \in C_{k}(k \in N) \text { with } x_{k} \rightarrow x\right\} .
\end{gathered}
$$

For a set-valued mapping $F: \mathbb{R}^{n} \rightarrow \mathbb{R}^{m}$ and $\bar{x} \in \mathbb{R}^{n}$, the outer and inner limit of $F$ as $x \rightarrow \bar{x}$ is naturally defined as 


$$
\begin{aligned}
& \operatorname{Limsup}_{x \rightarrow \bar{x}} F(x):=\left\{y \in \mathbb{R}^{m} \mid \exists x_{k} \rightarrow \bar{x}, y_{k} \rightarrow y \text { with } y_{k} \in F\left(x_{k}\right) \forall k \in \mathbb{N}\right\}, \\
& \underset{x \rightarrow \bar{x}}{\operatorname{Liminf}} F(x):=\left\{y \in \mathbb{R}^{m} \mid \forall x_{k} \rightarrow \bar{x}, \exists N \in \mathscr{N}_{\infty}, \exists y_{k} \rightarrow y \text { with } y_{k} \in F\left(x_{k}\right) \forall k \in N\right\} .
\end{aligned}
$$

Clearly, the inner limit is a subset of the outer limit. If they are equal, this set is called the Painlevé-Kuratowski limit and is denoted by $\operatorname{Lim}_{k \rightarrow \infty} C_{k}$, respectively $\operatorname{Lim}_{x \rightarrow \bar{x}} F(x)$.

Remark 2.1. Let $F(\cdot)$ be a uniformly bounded mapping defined in a neighborhood of the point $\bar{x} \in \mathbb{I}^{n}$ with nonempty images in a finite-dimensional space. It is easy to show that if the Painlevé-Kuratowski outer limit is a singleton Lim $\sup _{x \rightarrow \bar{x}} F(x)=\{\bar{y}\}$, it is equal to the Painlevé-Kuratowski limit. Indeed, by the assumption, for any sequence $x_{n} \rightarrow \bar{x}$, there is a converging subsequence $y_{n_{k}} \in F\left(x_{n_{k}}\right)$ and any such subsequence may have only the point $\bar{y}$ as the limit.

The classical Moreau-Rockafellar subdifferential of a convex function $f: \mathbb{R}^{n} \rightarrow \mathbb{R}$ at $x \in \mathbb{R}^{n}$ is

$$
\partial f(x):=\left\{s \in \mathbb{R}^{n} \mid \forall y \in \mathbb{R}^{n}:\langle s, y-x\rangle+f(x) \leq f(y)\right\} .
$$

It is well-known (see e.g. [15, Chap. V, Definition 1.1.4]) that

$$
\delta^{*}(l, \partial f(x))=f^{\prime}(x ; l),
$$

where $f^{\prime}(x ; l)$ is the directional derivative of $f$ at $x$ in direction $l$.

In the sequel, the Moreau-Rockafellar subdifferential of a sublinear function $g$ at zero is denoted by $\partial g$ instead of $\partial g(0)$.

Also, for the unique supporting point of a supporting face we denote

$$
d_{h}\left(l ; l^{\prime}\right)=y\left(l^{\prime}, Y(l, \partial h)\right) \quad\left(l, l^{\prime} \in \mathscr{S}_{1} \text { with } l \perp l^{\prime}\right) .
$$

The Dini subdifferential (see $[5,17,26,27]$ ) of a directionally differentiable function $f: \mathbb{R}^{n} \rightarrow \mathbb{R}$ at $x \in \mathbb{R}^{n}$ is

$$
\partial_{D} f(x)=\left\{v \in \mathbb{R}^{n} \mid f^{\prime}(x ; d) \geq\langle v, d\rangle \quad \forall d \in \mathbb{R}^{n}\right\} .
$$

The Fréchet subdifferential and the superdifferential/upper subdifferential (see [5, 6, 18, 23]) of a function $f: \mathbb{R}^{n} \rightarrow \mathbb{R}$ at a point $\bar{x} \in \mathbb{R}^{n}$ are defined as follows:

$$
\begin{aligned}
& \partial_{F} f(\bar{x})=\left\{v \in \mathbb{R}^{n} \mid \liminf _{x \rightarrow \bar{x}} \frac{f(x)-f(\bar{x})-\langle v, x-\bar{x}\rangle}{\|x-\bar{x}\|} \geq 0\right\}, \\
& \partial_{F}^{+} f(\bar{x})=\left\{v \in \mathbb{R}^{n} \mid \limsup _{x \rightarrow \bar{x}} \frac{f(x)-f(\bar{x})-\langle v, x-\bar{x}\rangle}{\| x-\bar{x} \mid} \leq 0\right\} .
\end{aligned}
$$

The Fréchet subdifferential coincides with the Fréchet gradient for a Fréchet differentiable function, and with the subdifferential for a convex function. One can think of $\partial_{F} f(\bar{x})$ and $\partial_{F}^{+} f(\bar{x})$ as of the set of gradients of linear functions "supporting" $f$ from below resp. above at $\bar{x}$. While the Fréchet subdifferential is defined for a vast class of functions, and can be used to check optimality conditions, in many cases it happens to be an empty set, which is a serious drawback for applications.

The Fréchet subdifferential possesses several useful properties summarized in the following two lemmas.

Lemma 2.2. Let $f: \mathbb{R}^{n} \rightarrow \mathbb{R}$ be positively homogeneous and $l \in \mathbb{R}^{n}$. Then

$$
\partial_{F} f(0)=\left\{v \in \mathbb{R}^{n} \mid f(d) \geq\langle v, d\rangle \quad \forall d \in \mathscr{S}_{n-1}\right\}
$$

and $f(\cdot)$ is the support function of the Fréchet subdifferential, i.e.

$$
f^{\prime}(0 ; l)=f(l) \text {. }
$$

Furthermore,

$$
\partial_{F} f(l)=\partial_{F} f(\lambda l), \quad \lambda>0
$$

Proof. The relation (6) is obtained easily from the positive homogeneity of $f$ and $f(0)=0$ (see e.g. [18, Proposition 1.9 a)]), and (8) follows from [18, Proposition 1.9 b)]. 
The following result, which is an immediate consequence of [9, Theorem 2], is used for evaluating Fréchet and Mordukhovich subdifferentials in the examples.

Lemma 2.3. Let $f: \mathbb{R}^{n} \rightarrow \mathbb{R}$ be directionally differentiable.

(i) If the directional derivative of $f$ at $x$ can be represented as

$$
f^{\prime}(x ; g)=\inf _{t \in T} \varphi_{t}(g)
$$

where $\varphi_{t}$ are sublinear functions for every $t \in T$ and $T$ is an arbitrary index set, then

$$
\partial_{F} f(x)=\bigcap_{t \in T} \partial \varphi_{t}(x)
$$

(ii) Analogously, if

$$
f^{\prime}(x ; g)=-\inf _{t \in T} \varphi_{t}(g),
$$

where $\varphi_{t}$ are sublinear functions for every $t \in T$, then

$$
\partial_{F}^{+} f(x)=\ominus \bigcap_{t \in T} \partial \varphi_{t}(x) .
$$

The next lemma states that the Fréchet subdifferential coincides with the Dini one for DC functions.

Lemma 2.4. If $f=g-h$ is DC with convex functions $g$ and $h$, then

$$
\partial_{F} f(x)=\partial_{D} f(x)=\left\{v \in \mathbb{R}^{n} \mid f^{\prime}(x ; l) \geq\langle v, l\rangle \quad \forall l \in \mathscr{S}_{n-1}\right\} .
$$

Proof. Since each convex function $g, h: \mathbb{R}^{n} \rightarrow \mathbb{R}$ is locally Lipschitz (see [15, Chap. IV, Theorem 3.1.2]), each DC function $f=g-h$ is also locally Lipschitz. Hence, we can apply Proposition 1.16 from [18], which yields

$$
d f(x)(l)=\liminf _{t \downarrow 0} \frac{f(x+t l)-f(x)}{t},
$$

where we use the notation in [18]. In our setting $d f(x)(l)$ corresponds to the lower Hadamard directional derivative of $f$ at $x$ in the direction $l$.

Since each convex function (and hence, each DC function) is directionally differentiable, the limit inferior is indeed a limit with $d f(x)(l)=f^{\prime}(x ; l)$. As we are dealing with finite-dimensional spaces, $d_{w} f(x ; l)=d f(x ; l)$ holds, and [18, Proposition 1.17] yields

$$
\begin{aligned}
\partial_{F} f(x) & =\left\{v \in \mathbb{R}^{n} \mid d_{w} f(x ; l) \geq\langle v, l\rangle \quad \forall l \in \mathbb{R}^{n}\right\} \\
& =\left\{v \in \mathbb{R}^{n} \mid f^{\prime}(x ; l) \geq\langle v, l\rangle \quad \forall l \in \mathbb{R}^{n}\right\}=\partial_{D} f(x) .
\end{aligned}
$$

Clearly, for convex functions it follows that

$$
\partial_{F} g(x)=\partial_{D} g(x)=\partial g(x)
$$

\section{The Mordukhovich and the Directed Subdifferential in $\mathbb{R}^{2}$}

For a continuous function $f: \mathbb{R}^{n} \rightarrow \mathbb{R}$, the Mordukhovich (lower) subdifferential and superdifferential (upper subdifferential) can be defined as a corresponding outer limit of Fréchet subdifferentials ([23, Theorem 1.89]):

$$
\begin{aligned}
& \partial_{M} f(\bar{x})=\operatorname{Limsup}_{x \rightarrow \bar{x}} \partial_{F} f(x), \\
& \partial_{M}^{+} f(\bar{x})=\operatorname{Limsup}_{x \rightarrow \bar{x}} \partial_{F}^{+} f(x) .
\end{aligned}
$$

The Mordukhovich symmetric subdifferential is defined as 


$$
\partial_{M}^{0} f(x)=\partial_{M} f(x) \cup \partial_{M}^{+} f(x)
$$

Here, the limits are in the Painlevé-Kuratowski sense. Furthermore, the connection between the Fréchet/Mordukhovich superdifferential to the corresponding subdifferential is given by the following formulas

$$
\partial_{F}^{+} f(\bar{x})=\ominus \partial_{F}(-f)(\bar{x}), \quad \partial_{M}^{+} f(\bar{x})=\ominus \partial_{M}(-f)(\bar{x}),
$$

which involve the negative function and the pointwise inverse of sets, see [18, remarks following Proposition 1.3] and [23, remarks below Definition 1.78].

Directed sets, offering a visualization of differences of two compact convex sets, are introduced and studied in $[2,3]$. Here, we only sketch the main ideas and notations on directed sets in $\mathbb{R}^{2}$.

The directed sets, as well as the embedding $J_{n}$ of convex compact sets in $\mathbb{R}^{n}$ into the Banach space of directed sets, are defined recursively in the space of dimension $n$. In one dimension, the directed embedded intervals are defined by the values of the support function in the two unit directions \pm 1 ,

$$
\overrightarrow{[a, b]}=J_{1}([a, b])=\left(\delta^{*}(\eta,[a, b])\right)_{\eta= \pm 1}=(-a, b) \quad(a \leq b)
$$

A general directed interval $\vec{A}_{1}=\overrightarrow{[c, d]}=(-c, d)$ allows that $c, d$ are arbitrary real numbers, even $c>d$ is possible (see references in [2,3]). A two-dimensional directed set $\vec{A}_{2}$ is a pair of a uniformly bounded map $\vec{A}_{1}(\cdot)$ having one-dimensional directed intervals [2] as its values (the directed "supporting face"), and a continuous function $a_{2}(\cdot): \mathbb{R}^{2} \rightarrow \mathbb{R}$ (the directed "support function"). This pair is parametrized by the unit vectors $l \in \mathbb{R}^{2}$ :

$$
\overrightarrow{A_{2}}=\left(\vec{A}_{1}(l), a_{2}(l)\right)_{l \in \mathscr{S}_{1}}
$$

A convex compact set $A \subset \mathbb{R}^{2}$ is embedded into the the space of two-dimensional directed sets via the embedding map $J_{2}$ composed from the natural projection $\pi_{1,2}$ from $\mathbb{R} \times\{0\} \subset \mathbb{R}^{2}$ onto $\mathbb{R}$, and the rotation $R_{2 l}$ which for any unit vector $l \in \mathbb{R}^{2}$ maps the pair $\left(l^{\prime}, l\right)$ (with $l^{\prime}$ orthonormal to $l$ ) to the standard basis $\left(e^{1}, e^{2}\right)$ in $\mathbb{R}^{2}$ :

$$
J_{2}(A)=\left(\overrightarrow{Y(l, A)}, \delta^{*}(l, A)\right)_{l \in \mathscr{S}_{1}}, \quad \text { with } \quad \overrightarrow{Y(l, A)}=J_{1}\left(\pi_{1,2} R_{2 l}\left(Y(l, A)-\delta^{*}(l, A) l\right)\right) .
$$

For a directed set $\vec{A}$, its visualization $V_{2}(\vec{A}) \subset \mathbb{R}^{2}$ has three parts - positive $P_{2}(\vec{A})$, negative $N_{2}(\vec{A})$ and mixedtype part $M_{2}(\vec{A})$ :

$$
\begin{aligned}
V_{2}(\vec{A}) & =P_{2}(\vec{A}) \cup N_{2}(\vec{A}) \cup M_{2}(\vec{A}) \\
M_{2}(\vec{A}) & =\bigcup_{l \in \mathscr{S}_{1}} Q_{2, l} V_{1}\left(\overrightarrow{A_{1}}(l)\right) \backslash\left(\partial P_{2}(\vec{A}) \cup \partial N_{2}(\vec{A})\right) .
\end{aligned}
$$

The last part is formed by reprojections $Q_{2, l}$ of one-dimensional visualizations from $\mathbb{R}$ onto the supporting lines $\langle x, l\rangle=a_{2}(l)$ for any unit vector $l \in \mathbb{R}^{2}$.

Equipped with a norm and operations acting separately on the components of the directed sets, the space of directed sets is a Banach space. The subtraction in this space is inverse to the Minkowski addition for embedded convex compact sets.

The directed subdifferential for DC functions and its visualization, the Rubinov subdifferential, are introduced in [4] for a DC function $f=g-h$ as

$$
\vec{\partial} f(x)=J_{2}(\partial g(x))-J_{2}(\partial h(x)), \quad \partial_{R} f(x)=V_{2}(\vec{\partial} f(x))
$$

i.e. it is the difference of the two embedded subdifferentials.

An explicit formula for the Mordukhovich subdifferential of a positively homogeneous function as a union of Fréchet subdifferentials is obtained in the next statement.

Proposition 3.1. Let $f: \mathbb{R}^{2} \rightarrow \mathbb{R}$ be a positively homogeneous function. Then

$$
\partial_{M} f(0)=\partial_{F} f(0) \cup \bigcup_{l \in \mathscr{S}_{1}}\left(\partial_{F} f(l) \cup \bigcup_{\substack{l^{\prime} \in \mathscr{S}_{1}, l \perp l^{\prime}}} \operatorname{Limsup} \partial_{F} f\left(l+t l^{\prime}\right)\right)
$$


Proof. Denote by $D$ the right-hand side of (20). We first show that $D \subseteq \partial_{M} f(0)$. Observe that $\partial_{F} f(0) \subset \partial_{M} f(0)$ holds by (13). Further, for any $l \in \mathscr{S}_{1}$ and $\lambda>0$ we have $\partial_{F} f(\lambda l)=\partial_{F} f(l)$ by Lemma 2.2 and

$$
\partial_{F} f(l)=\underset{\lambda \downarrow 0}{\operatorname{Limsup}} \partial_{F} f(\lambda l) \subset \underset{x \rightarrow 0}{\operatorname{Limsup}} \partial_{F} f(x)=\partial_{M} f(0) \text {. }
$$

It remains to show that for any $l, l^{\prime} \in \mathscr{S}_{1}, l \perp l^{\prime}$ we have

$$
\underset{t \downarrow 0}{\operatorname{Limsup}} \partial_{F} f\left(l+t l^{\prime}\right) \subset \partial_{M} f(0)
$$

Again, by Lemma 2.2 for any $t>0$

$$
\partial_{F} f\left(t\left(l+t l^{\prime}\right)\right)=\partial_{F} f\left(l+t l^{\prime}\right)
$$

Therefore,

$$
\underset{t \downarrow 0}{\operatorname{Limsup}} \partial_{F} f\left(l+t l^{\prime}\right)=\underset{t \downarrow 0}{\operatorname{Limsup}} \partial_{F} f\left(t\left(l+t l^{\prime}\right)\right) \subset \underset{x \rightarrow 0}{\operatorname{Limsup}} \partial_{F} f(x)=\partial_{M} f(0) .
$$

Now we will show that $\partial_{M} f(0) \subseteq D$. Let us consider an arbitrary element $v \in \partial_{M} f(0)$. By (13) there exist $\left\{v_{n}\right\}$ and $\left\{x_{n}\right\}$ such that $v_{n} \rightarrow v, x_{n} \rightarrow 0$ and $v_{n} \in \partial_{F} f\left(x_{n}\right)$. Without loss of generality, either $x_{n}=0$ for all $n$, or $x_{n} \neq 0$ for all $n$. In the former case, we have $v_{n} \in \partial_{F} f(0)$, and by the closedness of $\partial_{F} f(0)$

$$
v \in \underset{n \rightarrow \infty}{\operatorname{Limsup}} \partial_{F} f(0)=\partial_{F} f(0) \subset D
$$

In the latter case, without loss of generality suppose that $l_{n}=\frac{x_{n}}{\left\|x_{n}\right\|} \rightarrow l \in \mathscr{S}_{1}$. Observe that by Lemma 2.2

$$
\partial_{F} f\left(x_{n}\right)=\partial_{F} f\left(\frac{1}{\left\|x_{n}\right\|} x_{n}\right)=\partial_{F} f\left(l_{n}\right) .
$$

There are two possibilities again. Without loss of generality, either $l_{n}=l$ for all $n$, or $l_{n}-\left\langle l_{n}, l\right\rangle \cdot l \neq 0$ and $\left\langle l, l_{n}\right\rangle \neq 0$ for all $n$. In the first case, by (21)

$$
v \in \underset{n \rightarrow \infty}{\operatorname{Limsup}} \partial_{F} f\left(l_{n}\right)=\partial_{F} f(l) \subset D
$$

In the second case, let $l_{n}^{\prime}=\frac{l_{n}-\left\langle l_{n}, l\right\rangle \cdot l}{\left\|l_{n}-\left\langle l_{n}, l\right\rangle \cdot l\right\|}$ and $t_{n}=\frac{\left\|l_{n}-\left\langle l_{n}, l\right\rangle \cdot l\right\|}{\left\langle l_{n}, l\right\rangle}$. Observe that $l_{n}^{\prime} \perp l$, and $\left\|l_{n}^{\prime}\right\|=1$. Since in $\mathbb{R}^{2}$ there are only two unit vectors perpendicular to $l$, we can assume $l_{n}^{\prime}=l^{\prime} \in \mathscr{S}_{1}$ for all $n$, where $l^{\prime}$ is one of such two vectors. We have by (21) and Lemma 2.2

$$
v \in \underset{n \rightarrow \infty}{\operatorname{Limsup}} \partial_{F} f\left(\frac{l_{n}}{\left\langle l_{n}, l\right\rangle}\right)=\underset{n \rightarrow \infty}{\operatorname{Limsup}} \partial_{F} f\left(l+t_{n} l^{\prime}\right) \subset \underset{t \downarrow 0}{\operatorname{Limsup}} \partial_{F} f\left(l+t l^{\prime}\right) \subset D .
$$

The following result about the Fréchet subdifferential of a DC function follows from (11) and [14, Sect. 4] resp. [10, Chap. III, Proposition 4.1]. The following lemma will be used to explicitly calculate the first term appearing in the right-hand side of (20) in Proposition 3.1.

Lemma 3.2. Let $f=g-h$, where $g, h: \mathbb{R}^{n} \rightarrow \mathbb{R}$ are convex. Then

$$
\partial_{F} f(x)=\partial_{D} f(x)=\partial g(x) * \partial h(x),
$$

where $\partial g(x)$ and $\partial h(x)$ are the subdifferentials of $g$ and $h$ respectively.

To obtain a formula for the second term in the right-hand side of (20) for sublinear functions, we show now that the subdifferential of a sublinear function in a point $l \neq 0$ is a lower dimensional supporting face.

Lemma 3.3. Let $h: \mathbb{R}^{n} \rightarrow \mathbb{R}$ be convex. Then for any $l \in \mathbb{R}^{n}$,

$$
\partial\left[h^{\prime}(x ; \cdot)\right](l)=Y(l, \partial h(x)) .
$$

If, in addition, $h$ is sublinear, then

$$
\partial h(l)=Y(l, \partial h) .
$$


Proof. The equality (24) is trivial for $l=0$. It follows from [15, Chap. VI, Proposition 2.1.5] that for $l \neq 0$ and every convex function

$$
\partial\left[h^{\prime}(x ; \cdot)\right](l)=Y(l, \partial h(x))
$$

Setting $x=0$ the equality follows immediately, since (7) holds for the positively homogeneous function $h(\cdot)$.

In the next two lemmas we study the last term in the right-hand side of (20) for DS functions.

Lemma 3.4. Let $f=g-h$, where $g, h: \mathbb{R}^{2} \rightarrow \mathbb{R}$ are sublinear. Then for every $l, l^{\prime} \in \mathscr{S}_{1}$ with $l \perp l^{\prime}$,

$$
\underset{t \downarrow 0}{\operatorname{Limsup}} \partial_{F} f\left(l+t l^{\prime}\right) \neq \emptyset
$$

Proof. The function $f$ is locally Lipschitz as a difference of sublinear functions. Hence, $f$ is Fréchet differentiable almost everywhere, and there exists a sequence $\left\{x_{n}\right\}_{n} \subset \mathbb{R}^{2}$ such that $\left\langle x_{n}, l^{\prime}\right\rangle>0$ for all $n, x_{n} \rightarrow 0$ and $f$ is Fréchet differentiable at $l+x_{n}$. The Fréchet subdifferential of $f$ at $l+x_{n}$ is nonempty and coincides with the Fréchet derivative (see [18, Proposition 1.1]). Therefore, we have

$$
\partial_{F} f\left(l+x_{n}\right)=\left\{\nabla f\left(l+x_{n}\right)\right\} \quad(n \in \mathbb{N}) .
$$

Observe that for sufficiently large $n$ we have $1+\left\langle x_{n}, l\right\rangle>0$ and

$$
l+x_{n}=l+\left\langle x_{n}, l\right\rangle \cdot l+\left\langle x_{n}, l^{\prime}\right\rangle \cdot l^{\prime}=\left(1+\left\langle x_{n}, l\right\rangle\right)\left(l+\frac{\left\langle x_{n}, l^{\prime}\right\rangle}{1+\left\langle x_{n}, l\right\rangle} l^{\prime}\right) .
$$

The positive homogeneity of $f$ together with (8) yields

$$
\partial_{F} f\left(l+\frac{\left\langle x_{n}, l^{\prime}\right\rangle}{1+\left\langle x_{n}, l\right\rangle} l^{\prime}\right)=\partial_{F} f\left(l+x_{n}\right)=\left\{\nabla f\left(l+x_{n}\right)\right\} .
$$

Let $t_{n}=\frac{\left\langle x_{n}, l^{\prime}\right\rangle}{1+\left\langle x_{n}, l\right\rangle}$. Observe that $t_{n}>0$ and also $t_{n} \rightarrow 0$, i.e. $t_{n} \downarrow 0$. Since $f$ is locally Lipschitz, the sequence $\left\{\nabla f\left(l+x_{n}\right)\right\}$ is bounded, hence, has a converging subsequence. This subsequence satisfies

$$
\underset{n \rightarrow \infty}{\operatorname{Limsup}} \partial_{F} f\left(l+x_{n}\right)=\underset{n \rightarrow \infty}{\operatorname{Limsup}} \partial_{F} f\left(l+t_{n} l^{\prime}\right) \subset \underset{t \downarrow 0}{\operatorname{Limsup}} \partial_{F} f\left(l+t l^{\prime}\right)
$$

which yields the nonemptiness of $\operatorname{Limsup}_{t \downarrow 0} \partial_{F} f\left(l+t l^{\prime}\right)$.

The following result establishes that the set limit (i.e. the limit of the sequence) of the subdifferentials $\partial h(l+$ $\left.t l^{\prime}\right)$ evaluated at small orthogonal disturbances of the direction $l$ is a singleton. This fact is needed later in the representation theorem for directed subdifferentials.

Lemma 3.5. Let $h: \mathbb{R}^{2} \rightarrow \mathbb{R}$ be sublinear. Then for any $l, l^{\prime} \in \mathscr{S}_{1}$ with $l \perp l^{\prime}$, the set $Y\left(l^{\prime}, Y(l, \partial h)\right)$ is a singleton, and

$$
\operatorname{Lim}_{t \downarrow 0} \partial h\left(l+t l^{\prime}\right)=Y\left(l^{\prime}, Y(l, \partial h)\right)=\left\{y\left(l^{\prime}, Y(l, \partial h)\right)\right\}
$$

Proof. First, we will prove the claimed equality for the outer limit Limsup $t_{\downarrow} \partial h\left(l+t l^{\prime}\right)$, and then apply Remark 2.1 .

Let $\bar{v} \in Y(l, \partial h)$. Assume that $t_{n} \downarrow 0$ and $\left\{v_{n}\right\}_{n}$ is a sequence of points, each one in $\partial h\left(l+t_{n} l^{\prime}\right)$, and converging to a point in $\operatorname{Limsup}_{t \downarrow 0} \partial h\left(l+t l^{\prime}\right)$. Lemma 3.3 shows that

$$
v_{n} \in \partial h\left(l+t_{n} l^{\prime}\right)=Y\left(l+t_{n} l^{\prime}, \partial h\right) \quad(n \in \mathbb{N}) .
$$

By the definition of supporting face and by (7) we have

$$
\left\langle v_{n}, l+t_{n} l^{\prime}\right\rangle \geq\left\langle\bar{v}, l+t_{n} l^{\prime}\right\rangle=\langle\bar{v}, l\rangle+t_{n}\left\langle\bar{v}, l^{\prime}\right\rangle=h(l)+t_{n}\left\langle\bar{v}, l^{\prime}\right\rangle
$$

and

$$
\left\langle l, v_{n}\right\rangle \leq \delta^{*}\left(l, Y\left(l+t_{n} l^{\prime}, \partial h\right)\right) \leq \delta^{*}(l, \partial h)=h^{\prime}(0 ; l)=h(l) .
$$

Taking limits as $n \rightarrow \infty\left(t_{n} \downarrow 0\right)$ on both sides of (26) and (27), we obtain 


$$
\lim _{n \rightarrow \infty}\left\langle v_{n}, l\right\rangle=h(l) .
$$

Let $\tilde{v} \in Y\left(l^{\prime}, Y(l, \partial h)\right)$. Observe that $\tilde{v} \in Y(l, \partial h) \subset \partial h, v_{n} \in Y\left(l+t_{n} l^{\prime}, \partial h\right)$ and

$$
\begin{aligned}
& \left\langle v_{n}, l+t_{n} l^{\prime}\right\rangle=\left\langle v_{n}, l\right\rangle+t_{n}\left\langle v_{n}, l^{\prime}\right\rangle \leq\langle\tilde{v}, l\rangle+t_{n}\left\langle v_{n}, l^{\prime}\right\rangle, \\
& \left\langle v_{n}, l+t_{n} l^{\prime}\right\rangle \geq\left\langle\tilde{v}, l+t_{n} l^{\prime}\right\rangle=\langle\tilde{v}, l\rangle+t_{n}\left\langle\tilde{v}, l^{\prime}\right\rangle .
\end{aligned}
$$

Subtracting (30) from (29), we have $\left\langle v_{n}, l^{\prime}\right\rangle \geq\left\langle\tilde{v}, l^{\prime}\right\rangle$. Thus for any cluster point $\hat{v} \in \operatorname{Limsup}_{t \downarrow} \partial h\left(l+t l^{\prime}\right)$ of the sequence $\left\{v_{n}\right\}_{n}$, we have

$$
\left\langle\hat{v}, l^{\prime}\right\rangle \geq\left\langle\tilde{v}, l^{\prime}\right\rangle .
$$

Since $Y(\cdot, \partial h)$ is upper semicontinuous and has closed values, it follows from (24) and $v_{n} \in Y\left(l+t_{n} l^{\prime}, \partial h\right)$ that $\hat{v} \in Y(l, \partial h)$. Hence, $\hat{v} \in Y\left(l^{\prime}, Y(l, \partial h)\right)$ by (31) and the inclusion " $\subset$ " in (25) is proved with the outer limit in the left-hand side.

Assume now that $Y\left(l^{\prime}, Y(l, \partial h)\right)$ contains two different points $\tilde{v}_{1}, \tilde{v}_{2}$. Clearly,

$$
\begin{aligned}
\left\langle l^{\prime}, \tilde{v}_{1}\right\rangle & =\left\langle l^{\prime}, \tilde{v}_{2}\right\rangle=\delta^{*}\left(l^{\prime}, Y(l, \partial h)\right), \\
\left\langle l, \tilde{v}_{1}\right\rangle & =\left\langle l, \tilde{v}_{2}\right\rangle=\delta^{*}(l, \partial h) .
\end{aligned}
$$

For any $\eta \in \mathbb{R}^{2}$ the representation $\eta=\langle\eta, l\rangle \cdot l+\left\langle\eta, l^{\prime}\right\rangle \cdot l^{\prime}$ is valid, therefore

$$
\left\langle\eta, \tilde{v}_{1}-\tilde{v}_{2}\right\rangle=\langle\eta, l\rangle \cdot\left\langle l, \tilde{v}_{1}-\tilde{v}_{2}\right\rangle+\left\langle\eta, l^{\prime}\right\rangle \cdot\left\langle l^{\prime}, \tilde{v}_{1}-\tilde{v}_{2}\right\rangle=0,
$$

which contradicts the assumption that the points are different.

Hence, the right-hand side in (25) is just a singleton and the equality follows by the non-emptiness of the left-hand side guaranteed by Lemma 3.4, Equ. (12) and Remark 2.1.

Thus, (25) in the above lemma can be reformulated with the notation (5) as

$$
\operatorname{Lim}_{t \downarrow 0} \partial h\left(l+t l^{\prime}\right)=\left\{d_{h}\left(l ; l^{\prime}\right)\right\} .
$$

The previous lemma will be generalized to DC functions. The following lemma states an explicit formula for the third term appearing in the right-hand side of (20) in Proposition 3.1.

Lemma 3.6. Let $f=g-h$, where $g, h: \mathbb{R}^{2} \rightarrow \mathbb{R}$ are sublinear. Then for every $l, l^{\prime} \in \mathscr{S}_{1}, l^{\prime} \perp l$ the outer limit Limsup $\operatorname{st}_{t \downarrow} \partial_{F} f\left(l+t l^{\prime}\right)$ is a singleton, and

$$
\underset{t \downarrow 0}{\operatorname{Limsup}} \partial_{F} f\left(l+t l^{\prime}\right)=\left\{y\left(l^{\prime}, Y(l, \partial g)\right)-y\left(l^{\prime}, Y(l, \partial h)\right)\right\} .
$$

Proof. Let

$$
u \in \underset{t \downarrow 0}{\operatorname{Limsup}} \partial_{F} f\left(l+t l^{\prime}\right) .
$$

Then there exist sequences $\left\{u_{n}\right\},\left\{t_{n}\right\}, u_{n} \rightarrow u, t_{n} \downarrow 0$ such that $u_{n} \in \partial_{F} f\left(l+t_{n} l^{\prime}\right)$. By Lemma 3.2 we have

$$
\partial_{F} f\left(l+t_{n} l^{\prime}\right)=\partial g\left(l+t_{n} l^{\prime}\right) * \partial h\left(l+t_{n} l^{\prime}\right) \quad(n \in \mathbb{N}) .
$$

Therefore, for all $n \in \mathbb{N}$ there are

$$
v_{n} \in \partial g\left(l+t_{n} l^{\prime}\right) \quad \text { and } \quad w_{n} \in \partial h\left(l+t_{n} l^{\prime}\right)
$$

such that $u_{n}=v_{n}-w_{n}$.

Since $\left\{v_{n}\right\}$ and $\left\{w_{n}\right\}$ are bounded (as they belong to the corresponding upper semicontinuous subdifferentials of $g$ and $h$ ), the sets $\operatorname{Limsup}_{n \rightarrow \infty}\left\{v_{n}\right\}$ and $\operatorname{Limsup}_{n \rightarrow \infty}\left\{w_{n}\right\}$ of cluster points of the corresponding sequences are nonempty. Moreover, by Lemma 3.5 we have

$$
\begin{aligned}
& \operatorname{Limsup}_{n \rightarrow \infty}\left\{v_{n}\right\} \subset \underset{n \rightarrow \infty}{\operatorname{Limsup}} \partial g\left(l+t_{n} l^{\prime}\right)=\operatorname{Lim}_{n \rightarrow \infty} \partial g\left(l+t_{n} l^{\prime}\right)=\left\{d_{g}\left(l ; l^{\prime}\right)\right\}, \\
& \underset{n \rightarrow \infty}{\operatorname{Limsup}}\left\{w_{n}\right\} \subset \underset{n \rightarrow \infty}{\operatorname{Limsup}} \partial h\left(l+t_{n} l^{\prime}\right)=\operatorname{Lim}_{n \rightarrow \infty} \partial h\left(l+t_{n} l^{\prime}\right)=\left\{d_{h}\left(l ; l^{\prime}\right)\right\},
\end{aligned}
$$


where we have used the notation (5).

Hence the sequences $\left\{v_{n}\right\}$ and $\left\{w_{n}\right\}$ converge and have unique cluster points. Therefore

$$
u=\lim _{n \rightarrow \infty} u_{n}=\lim _{n \rightarrow \infty} v_{n}-\lim _{n \rightarrow \infty} w_{n}=d_{g}\left(l ; l^{\prime}\right)-d_{h}\left(l ; l^{\prime}\right) .
$$

Since $u$ is arbitrary, we have

$$
\underset{t \downarrow 0}{\operatorname{Limsup}} \partial_{F} f\left(l+t l^{\prime}\right) \subset\left\{d_{g}\left(l ; l^{\prime}\right)-d_{h}\left(l ; l^{\prime}\right)\right\}
$$

Applying Lemma 3.4,

$$
\underset{t \downarrow 0}{\operatorname{Limsup}} \partial_{F} f\left(l+t l^{\prime}\right) \neq \emptyset
$$

holds and we obtain (33) from (34).

For the convenience of the reader, we include a full proof for the explicit formula of the subdifferential of a sublinear function with the help of two collinear directions orthogonal to the supporting face in Lemma 3.3, although this geometric fact is rather obvious.

Lemma 3.7. Let $h: \mathbb{R}^{2} \rightarrow \mathbb{R}$ be a sublinear function. Then for every $l, l^{\prime} \in \mathscr{S}_{1}$ with $l^{\prime} \perp l$,

$$
\partial h(l)=\operatorname{co}\left\{d_{h}\left(l ;-l^{\prime}\right), d_{h}\left(l ; l^{\prime}\right)\right\},
$$

where we used again the notation (5).

Proof. From Lemma 3.3 we know that

$$
\partial h(l)=Y(l, \partial h) .
$$

Obviously, $\operatorname{co}\left\{d_{h}\left(l ;-l^{\prime}\right), d_{h}\left(l ; l^{\prime}\right)\right\} \subset Y(l, \partial h)$, and we only need to show the opposite inclusion. Assume that there exists $v \in Y(l, \partial h)$ such that

$$
v \notin \operatorname{co}\left\{d_{h}\left(l ;-l^{\prime}\right), d_{h}\left(l ; l^{\prime}\right)\right\} .
$$

Then by the separation theorem there exists $\tilde{l} \in \mathbb{R}^{2}$ such that

$$
\langle v, \tilde{l}\rangle>\max \left\{\left\langle d_{h}\left(l ;-l^{\prime}\right), \tilde{l}\right\rangle,\left\langle d_{h}\left(l ; l^{\prime}\right), \tilde{l}\right\rangle\right\}
$$

Since the representation $v=\langle v, l\rangle \cdot l+\left\langle v, l^{\prime}\right\rangle \cdot l^{\prime}$ holds, we can use $v \in Y(l, \partial h)$ as well as (4) and (7) to observe that

$$
\langle\tilde{l}, v\rangle=\langle\tilde{l}, l\rangle \cdot\langle v, l\rangle+\left\langle\tilde{l}, l^{\prime}\right\rangle \cdot\left\langle v, l^{\prime}\right\rangle=\langle\tilde{l}, l\rangle \cdot h(l)+\left\langle\tilde{l}, l^{\prime}\right\rangle \cdot\left\langle v, l^{\prime}\right\rangle \text {. }
$$

Using $d_{h}\left(l ; l^{\prime}\right) \in Y(l, \partial h)$ twice, the equality $h(l)=\left\langle d_{h}\left(l, l^{\prime}\right), l\right\rangle$ follows, if $\left\langle\tilde{l}, l^{\prime}\right\rangle \geq 0$, as well as

$$
\langle\tilde{l}, v\rangle \leq\langle\tilde{l}, l\rangle \cdot h(l)+\left\langle\tilde{l}, l^{\prime}\right\rangle \cdot\left\langle d_{h}\left(l ; l^{\prime}\right), l^{\prime}\right\rangle=\left\langle d_{h}\left(l ; l^{\prime}\right), \tilde{l}\right\rangle \leq \max \left\{\left\langle d_{h}\left(l ;-l^{\prime}\right), \tilde{l}\right\rangle,\left\langle d_{h}\left(l ; l^{\prime}\right), \tilde{l}\right\rangle\right\} .
$$

Analogously, if $\left\langle\tilde{l}, l^{\prime}\right\rangle<0$, the following estimate is valid due to $h(l)=\left\langle d_{h}\left(l,-l^{\prime}\right), l\right\rangle$ :

$$
\langle\tilde{l}, v\rangle \leq\langle\tilde{l}, l\rangle \cdot h(l)-\left\langle\tilde{l}, l^{\prime}\right\rangle \cdot\left\langle d_{h}\left(l ;-l^{\prime}\right),-l^{\prime}\right\rangle=\left\langle d_{h}\left(l ;-l^{\prime}\right), \tilde{l}\right\rangle \leq \max \left\{\left\langle d_{h}\left(l ;-l^{\prime}\right), \tilde{l}\right\rangle,\left\langle d_{h}\left(l ; l^{\prime}\right), \tilde{l}\right\rangle\right\}
$$

Clearly, (36) resp. (37) contradict (35), hence our assumption is wrong.

The next two lemmas will be used in the further theorems. The first one connects the first component of the embedding (17) of convex sets into the space of directed sets to the interval which coincides with the projection of the line segment from Lemma 3.7. In the embedding the natural projection $\pi_{1,2}$ and the rotation $R_{2, l}$ in [2] are used.

Lemma 3.8. Let $h: \mathbb{R}^{2} \rightarrow \mathbb{R}$ be sublinear, $l \in \mathscr{S}_{1}$ and $l^{\prime}=R_{2, l}^{\top} e^{1}$. Then, the embedding in (17) satisfies

$$
\pi_{1,2} R_{2, l}(Y(l, \partial h)-h(l) l)=\left[\left\langle d_{h}\left(l ;-l^{\prime}\right), l^{\prime}\right\rangle,\left\langle d_{h}\left(l ; l^{\prime}\right), l^{\prime}\right\rangle\right]
$$

where we used again the notation (5).

Proof. Observe that $l \perp l^{\prime}$, so that Lemmas 3.3 and 3.7 apply with

$$
Y(l, \partial h)=\operatorname{co}\left\{d_{h}\left(l ;-l^{\prime}\right), d_{h}\left(l ; l^{\prime}\right)\right\} .
$$


Since $h(l)=\left\langle d_{h}\left(l ; \pm l^{\prime}\right), l\right\rangle$, the following representation holds:

$$
d_{h}\left(l ; \pm l^{\prime}\right)=\left\langle d_{h}\left(l ; \pm l^{\prime}\right), l^{\prime}\right\rangle \cdot l^{\prime}+\left\langle d_{h}\left(l ; \pm l^{\prime}\right), l\right\rangle \cdot l=\left\langle d_{h}\left(l ; \pm l^{\prime}\right), l^{\prime}\right\rangle \cdot l^{\prime}+h(l) l
$$

Therefore,

$$
\begin{aligned}
\pi_{1,2} R_{2, l}(Y(l, \partial h)-h(l) l) & =\pi_{1,2} R_{2, l}\left(\operatorname{co}\left\{d_{h}\left(l ;-l^{\prime}\right), d_{h}\left(l ; l^{\prime}\right)\right\}-h(l) l\right) \quad(\text { by (38)) } \\
& =\pi_{1,2} R_{2, l}\left(\operatorname{co}\left\{d_{h}\left(l ;-l^{\prime}\right)-h(l) l, d_{h}\left(l ; l^{\prime}\right)-h(l) l\right\}\right) \\
& =\pi_{1,2} R_{2, l}\left(\operatorname{co}\left\{\left\langle d_{h}\left(l ;-l^{\prime}\right), l^{\prime}\right\rangle l^{\prime},\left\langle d_{h}\left(l ; l^{\prime}\right), l^{\prime}\right\rangle l^{\prime}\right\}\right) \quad \text { (by (39)) } \\
& =\operatorname{co}\left\{\pi_{1,2} R_{2, l}\left\langle d_{h}\left(l ;-l^{\prime}\right), l^{\prime}\right\rangle l^{\prime}, \pi_{1,2} R_{2, l}\left(\left\langle d_{h}\left(l ; l^{\prime}\right), l^{\prime}\right\rangle l^{\prime}\right\}\right. \\
& =\operatorname{co}\left\{\left\langle d_{h}\left(l ;-l^{\prime}\right), l^{\prime}\right\rangle \cdot \pi_{1,2} R_{2, l} l^{\prime},\left\langle d_{h}\left(l ; l^{\prime}\right), l^{\prime}\right\rangle \cdot \pi_{1,2} R_{2, l} l^{\prime}\right\} \\
& =\operatorname{co}\left\{\left\langle d_{h}\left(l ;-l^{\prime}\right), l^{\prime}\right\rangle,\left\langle d_{h}\left(l ; l^{\prime}\right), l^{\prime}\right\rangle\right\} \\
& =\left[\left\langle d_{h}\left(l ;-l^{\prime}\right), l^{\prime}\right\rangle,\left\langle d_{h}\left(l ; l^{\prime}\right), l^{\prime}\right\rangle\right] \quad\left(\text { as }\left\langle d_{h}\left(l ;-l^{\prime}\right), l^{\prime}\right\rangle \leq\left\langle d_{h}\left(l ; l^{\prime}\right), l^{\prime}\right\rangle\right) .
\end{aligned}
$$

The following lemma generalizes Lemma 3.8 to DS functions. To study the result of the embedded difference of subdifferentials, the convex sets in the first component of the embedding (17) can be calculated with the help of the two endpoints of the interval.

Lemma 3.9. Let $f=g-h$, where $g, h: \mathbb{R}^{2} \rightarrow \mathbb{R}$ are sublinear. Consider $l \in \mathscr{S}_{1}$ and the orthogonal vector $l^{\prime}=$ $R_{2, l}^{\top} e^{1}$. Then

$$
\begin{aligned}
& \pi_{1,2} R_{2, l}\left(D^{-}(l)-f(l) l\right)=\left\langle d_{g}\left(l ;-l^{\prime}\right)-d_{h}\left(l ;-l^{\prime}\right), l^{\prime}\right\rangle, \\
& \pi_{1,2} R_{2, l}\left(D^{+}(l)-f(l) l\right)=\left\langle d_{g}\left(l ; l^{\prime}\right)-d_{h}\left(l ; l^{\prime}\right), l^{\prime}\right\rangle,
\end{aligned}
$$

where the notation (5) and

$$
D^{-}(l):=\underset{t \downarrow 0}{\operatorname{Limsup}} \partial_{F} f\left(l-t l^{\prime}\right) \quad \text { and } \quad D^{+}(l):=\underset{t \downarrow 0}{\operatorname{Limsup}} \partial_{F} f\left(l+t l^{\prime}\right)
$$

are used.

Proof. Clearly, $l \perp l^{\prime}$. By Lemma 3.6 the two sets

$$
D^{-}(l)=d_{g}\left(l ;-l^{\prime}\right)-d_{h}\left(l ;-l^{\prime}\right), \quad D^{+}(l)=d_{g}\left(l ; l^{\prime}\right)-d_{h}\left(l ; l^{\prime}\right)
$$

are singletons and $d_{g}\left(l ; \pm l^{\prime}\right) \in Y(l, \partial g), d_{h}\left(l ; \pm l^{\prime}\right) \in Y(l, \partial h)$. Therefore,

$$
\begin{aligned}
\pi_{1,2} R_{2, l}\left(D^{-}(l)-f(l) l\right)= & \pi_{1,2} R_{2, l}\left(D^{-}(l)-f(l) l\right) \\
= & \pi_{1,2} R_{2, l}\left(d_{g}\left(l ;-l^{\prime}\right)-d_{h}\left(l ;-l^{\prime}\right)-f(l) l\right) \\
= & \pi_{1,2} R_{2, l}\left(\left\langle d_{g}\left(l ;-l^{\prime}\right)-d_{h}\left(l ;-l^{\prime}\right), l^{\prime}\right\rangle l^{\prime}\right. \\
& \left.+\left\langle d_{g}\left(l ;-l^{\prime}\right)-d_{h}\left(l ;-l^{\prime}\right), l\right\rangle l-f(l) l\right) \\
= & \pi_{1,2} R_{2, l}\left(\left\langle d_{g}\left(l ;-l^{\prime}\right)-d_{h}\left(l ;-l^{\prime}\right), l^{\prime}\right\rangle l^{\prime}+(g(l)-h(l)) l-f(l) l\right) \\
= & \pi_{1,2} R_{2, l}\left(\left\langle d_{g}\left(l ;-l^{\prime}\right)-d_{h}\left(l ;-l^{\prime}\right), l^{\prime}\right\rangle l^{\prime}\right) \\
= & \left\langle d_{g}\left(l ;-l^{\prime}\right)-d_{h}\left(l ;-l^{\prime}\right), l^{\prime}\right\rangle \cdot \pi_{1,2} R_{2, l} l^{\prime} \\
= & \left\langle d_{g}\left(l ;-l^{\prime}\right)-d_{h}\left(l ;-l^{\prime}\right), l^{\prime}\right\rangle \cdot \pi_{1,2} R_{2, l} R_{2, l}^{\top} l^{1} \\
= & \left\langle d_{g}\left(l ;-l^{\prime}\right)-d_{h}\left(l ;-l^{\prime}\right), l^{\prime}\right\rangle
\end{aligned}
$$

and analogously

$$
\pi_{1,2} R_{2, l}\left(D^{+}(l)-f(l) l\right)=\left\langle d_{g}\left(l ; l^{\prime}\right)-d_{h}\left(l ; l^{\prime}\right), l^{\prime}\right\rangle
$$

We apply the two lemmas above to represent the directed subdifferential of a positively homogeneous DC function in $\mathbb{R}^{2}$ with the help of outer limits of Fréchet subdifferential. The unique supporting points calculated in Lemma 3.9 are used to determine the (one-dimensional) first component of the directed subdifferential. 
Lemma 3.10. $f=g-h, g, h: \mathbb{R}^{2} \rightarrow \mathbb{R}$, sublinear. Then, using the notation (5),

$$
\vec{\partial} f(0)=\left(\overrightarrow{\left[\left\langle d_{g}\left(l ;-l^{\prime}\right)-d_{h}\left(l ;-l^{\prime}\right), l^{\prime}\right\rangle,\left\langle d_{g}\left(l ; l^{\prime}\right)-d_{h}\left(l ; l^{\prime}\right), l^{\prime}\right\rangle\right]}, f(l)\right)_{l \in \mathscr{S}_{1}}
$$

with $l^{\prime}=l^{\prime}(l)=R_{2, l}^{\top} e^{1}$.

Proof. Observe that $\delta^{*}(l, \partial g)=g^{\prime}(0 ; l)=g(l)$ by (4) and Lemma 2.2 and therefore,

$$
\begin{aligned}
& \vec{\partial} f=J_{2}(\partial g)-J_{2}(\partial h) \quad \text { (by definition) } \\
& =\left(J_{1}\left(\pi_{1,2} R_{2, l}(Y(l, \partial g)-g(l) l)\right), g(l)\right)_{l \in \mathscr{S}_{1}} \\
& -\left(J_{1}\left(\pi_{1,2} R_{2, l}(Y(l, \partial h)-h(l) l)\right), h(l)\right)_{l \in \mathscr{S}_{1}} \quad \text { (by definition) } \\
& =\left(J_{1}\left(\left[\left\langle d_{g}\left(l ;-l^{\prime}\right), l^{\prime}\right\rangle,\left\langle d_{g}\left(l ; l^{\prime}\right), l^{\prime}\right\rangle\right]\right)-J_{1}\left(\left[\left\langle d_{h}\left(l ;-l^{\prime}\right), l^{\prime}\right\rangle,\left\langle d_{h}\left(l ; l^{\prime}\right), l^{\prime}\right\rangle\right]\right), g(l)-h(l)\right)_{l \in \mathscr{S}_{1}} \\
& \text { (by Lemma 3.8) } \\
& =\left(\overrightarrow{\left[\left\langle d_{g}\left(l ;-l^{\prime}\right), l^{\prime}\right\rangle,\left\langle d_{g}\left(l ; l^{\prime}\right), l^{\prime}\right\rangle\right]}-\overrightarrow{\left[\left\langle d_{h}\left(l ;-l^{\prime}\right), l^{\prime}\right\rangle,\left\langle d_{h}\left(l ; l^{\prime}\right), l^{\prime}\right\rangle\right]}, f(l)\right)_{l \in \mathscr{S}_{1}} \\
& =\left(\overrightarrow{\left[\left\langle d_{g}\left(l ;-l^{\prime}\right), l^{\prime}\right\rangle-\left\langle d_{h}\left(l ;-l^{\prime}\right), l^{\prime}\right\rangle,\left\langle d_{g}\left(l ; l^{\prime}\right), l^{\prime}\right\rangle-\left\langle d_{h}\left(l ; l^{\prime}\right), l^{\prime}\right\rangle\right]}, f(l)\right)_{l \in \mathscr{S}_{1}} .
\end{aligned}
$$

As a first main result, we connect the representation of the directed subdifferential to outer limits of Fréchet subdifferentials.

Theorem 3.11. Let $g, h: \mathbb{R}^{2} \rightarrow \mathbb{R}$ be sublinear functions, and let $f=g-h$. Then the directed subdifferential of $f$ at zero $\vec{A}=\left(\overrightarrow{A_{1}}(l), a_{2}(l)\right)_{l \in \mathscr{S}_{1}}$ can be constructed via limits of Fréchet normals as follows: for every $l \in \mathscr{S}_{1}$ let

$$
f_{2}(l):=f(l), \quad \overrightarrow{F_{1}}(l):=\overrightarrow{\left[\pi_{1,2} R_{2, l}\left(D^{-}(l)-f(l) l\right), \pi_{1,2} R_{2, l}\left(D^{+}(l)-f(l) l\right)\right]}
$$

where $D^{-}(l):=\operatorname{Limsup}_{t \downarrow 0} \partial_{F} f\left(l-t l^{\prime}\right), \quad D^{+}(l):=\operatorname{Limsup}_{t \downarrow 0} \partial_{F} f\left(l+t l^{\prime}\right)$, and $l^{\prime}:=R_{2, l}^{\top} e^{1}$. Then, $\vec{F}=\left(\overrightarrow{F_{1}}(l), f_{2}(l)\right)_{l \in \mathscr{S}_{1}}$ coincides with $\vec{A}=\vec{\partial} f(0)$.

Proof. By Lemma 3.9

$$
\begin{aligned}
& \pi_{1,2} R_{2, l}\left(D^{-}(l)-f(l) l\right)=\left\langle d_{g}\left(l ;-l^{\prime}\right)-d_{h}\left(l ;-l^{\prime}\right), l^{\prime}\right\rangle \\
& \pi_{1,2} R_{2, l}\left(D^{+}(l)-f(l) l\right)=\left\langle d_{g}\left(l ; l^{\prime}\right)-d_{h}\left(l ; l^{\prime}\right), l^{\prime}\right\rangle
\end{aligned}
$$

where we used again the notation (5). Therefore,

$$
\left.\vec{F}=\left(\overrightarrow{\left\langle\left\langle d_{g}\left(l ;-l^{\prime}\right)-d_{h}\left(l ;-l^{\prime}\right), l^{\prime}\right\rangle,\left\langle d_{g}\left(l ; l^{\prime}\right)-d_{h}\left(l ; l^{\prime}\right), l^{\prime}\right\rangle\right.}\right], f(l)\right)_{l \in \mathscr{S}_{1}}
$$

which coincides with the directed subdifferential $\vec{A}$ of $f$ by Lemma 3.10.

The equality for the Fréchet subdifferential in the next lemma will be used to explicitly calculate the second term appearing in the right-hand side of (20) in Proposition 3.1. Geometrically, this fact is easy to believe so the reader may skip the technical proof.

Lemma 3.12. Let $f=g-h$, where $g, h: \mathbb{R}^{2} \rightarrow \mathbb{R}$ are sublinear. Then for every $l \in \mathscr{S}_{1}$

$$
\begin{aligned}
\partial_{F} f(l) & =\partial g(l) * \partial h(l)=\operatorname{co}\left\{d_{g}\left(l ;-l^{\prime}\right), d_{g}\left(l ; l^{\prime}\right)\right\} * \operatorname{co}\left\{d_{h}\left(l ;-l^{\prime}\right), d_{h}\left(l ; l^{\prime}\right)\right\} \\
& =\left\{\begin{array}{cc}
\operatorname{co}\left\{d_{g}\left(l ;-l^{\prime}\right)-d_{h}\left(l ;-l^{\prime}\right), d_{g}\left(l ; l^{\prime}\right)-d_{h}\left(l ; l^{\prime}\right)\right\}, & \text { if case 1 holds, } \\
\emptyset, & \text { if case } 2 \text { holds, }
\end{array}\right.
\end{aligned}
$$

where we used again the notation (5) and $l^{\prime}=R_{2, l}^{\top} e^{1}$. Case 1 holds, if

$$
\left\langle d_{g}\left(l ;-l^{\prime}\right)-d_{h}\left(l ;-l^{\prime}\right), l^{\prime}\right\rangle \leq\left\langle d_{g}\left(l ; l^{\prime}\right)-d_{h}\left(l ; l^{\prime}\right), l^{\prime}\right\rangle
$$

and case 2 is given, if the inequality "> " holds. 
Proof. Lemmas 3.3 and 3.7 show that

$$
\begin{aligned}
& \partial g(l)=Y(l, \partial g)=\operatorname{co}\left\{d_{g}\left(l ;-l^{\prime}\right), d_{g}\left(l ; l^{\prime}\right)\right\}, \\
& \partial h(l)=Y(l, \partial h)=\operatorname{co}\left\{d_{h}\left(l ;-l^{\prime}\right), d_{h}\left(l ; l^{\prime}\right)\right\},
\end{aligned}
$$

since $l \perp l^{\prime}$. Clearly, for all $v \in \partial g(l)$ and $w \in \partial h(l)$, (4) and (7) apply, i.e.

$$
\begin{aligned}
\langle l, v\rangle & =\delta^{*}(l, \partial g)=g^{\prime}(0 ; l)=g(l), \\
\langle l, w\rangle & =\delta^{*}(l, \partial h)=h^{\prime}(0 ; l)=h(l)
\end{aligned}
$$

and especially,

$$
\left\langle l, d_{g}\left(l ; \pm l^{\prime}\right)\right\rangle=g(l), \quad\left\langle l, d_{h}\left(l ; \pm l^{\prime}\right)\right\rangle=h(l)
$$

It holds that

$$
\begin{aligned}
\partial g(l) & =\operatorname{co}\left\{d_{g}\left(l ;-l^{\prime}\right)-g(l) l, d_{g}\left(l ; l^{\prime}\right)-g(l) l\right\}+g(l) l \\
& =\operatorname{co}\left\{\left\langle d_{g}\left(l ;-l^{\prime}\right), l^{\prime}\right\rangle \cdot l^{\prime},\left\langle d_{g}\left(l ; l^{\prime}\right), l^{\prime}\right\rangle \cdot l^{\prime}\right\}+g(l) l, \\
\partial h(l) & =\operatorname{co}\left\{d_{h}\left(l ;-l^{\prime}\right)-h(l) l, d_{h}\left(l ; l^{\prime}\right)-h(l) l\right\}+h(l) l \\
& =\operatorname{co}\left\{\left\langle d_{h}\left(l ;-l^{\prime}\right), l^{\prime}\right\rangle \cdot l^{\prime},\left\langle d_{h}\left(l ; l^{\prime}\right), l^{\prime}\right\rangle \cdot l^{\prime}\right\}+h(l) l, \\
\partial g(l)-g(l) l & =\operatorname{co}\left\{\left\langle d_{g}\left(l ;-l^{\prime}\right), l^{\prime}\right\rangle \cdot l^{\prime},\left\langle d_{g}\left(l ; l^{\prime}\right), l^{\prime}\right\rangle \cdot l^{\prime}\right\}, \\
\partial h(l)-h(l) l & =\operatorname{co}\left\{\left\langle d_{h}\left(l ;-l^{\prime}\right), l^{\prime}\right\rangle \cdot l^{\prime},\left\langle d_{h}\left(l ; l^{\prime}\right), l^{\prime}\right\rangle \cdot l^{\prime}\right\} .
\end{aligned}
$$

Let us denote for abbreviation

$$
\begin{array}{ll}
\mu_{1}:=\left\langle d_{g}\left(l ;-l^{\prime}\right), l^{\prime}\right\rangle, & \mu_{2}:=\left\langle d_{g}\left(l ; l^{\prime}\right), l^{\prime}\right\rangle, \\
v_{1}:=\left\langle d_{h}\left(l ;-l^{\prime}\right), l^{\prime}\right\rangle, & v_{2}:=\left\langle d_{h}\left(l ; l^{\prime}\right), l^{\prime}\right\rangle .
\end{array}
$$

Since $d_{g}\left(l ; l^{\prime}\right) \in y\left(l^{\prime}, Y(l, \partial g)\right)$ and $d_{h}\left(l ; l^{\prime}\right) \in y\left(l^{\prime}, Y(l, \partial h)\right)$, we have the ordering

$$
\mu_{1} \leq \mu_{2} \quad \text { and } \quad v_{1} \leq v_{2} .
$$

Let us study the scalar product of $u \in(\partial g(l)-g(l) l) * *(\partial h(l)-h(l) l)$ and $\eta \in \mathbb{R}^{n}$ :

$$
\begin{aligned}
\langle\eta, u\rangle & \leq \delta^{*}\left(\eta, \operatorname{co}\left\{\mu_{1} l^{\prime}, \mu_{2} l^{\prime}\right\}\right)-\delta^{*}\left(\eta, \operatorname{co}\left\{v_{1} l^{\prime}, v_{2} l^{\prime}\right\}\right) \\
& =\max \left\{\left\langle\eta, \mu_{1} l^{\prime}\right\rangle,\left\langle\eta, \mu_{2} l^{\prime}\right\rangle\right\}-\max \left\{\left\langle\eta, v_{1} l^{\prime}\right\rangle,\left\langle\eta, v_{2} l^{\prime}\right\rangle\right\} \\
& =\max \left\{\mu_{1} \cdot\left\langle\eta, l^{\prime}\right\rangle, \mu_{2} \cdot\left\langle\eta, l^{\prime}\right\rangle\right\}-\max \left\{v_{1} \cdot\left\langle\eta, l^{\prime}\right\rangle, v_{2} \cdot\left\langle\eta, l^{\prime}\right\rangle\right\}
\end{aligned}
$$

Both shifted line segments are spanned by the vector $l^{\prime}$, hence the geometric difference lies also in this span which is demonstrated by setting $\eta= \pm l$ in the above inequality:

$$
\langle l, u\rangle \leq 0-0=0, \quad\langle-l, u\rangle \leq 0-0=0
$$

Hence, $\langle l, u\rangle=0$. Let us study the scalar product in the orthogonal directions $l^{\prime}$ and $-l^{\prime}$.

$$
\begin{aligned}
\left\langle l^{\prime}, u\right\rangle & \leq \max \left\{\mu_{1}, \mu_{2}\right\}-\max \left\{v_{1}, v_{2}\right\}=\mu_{2}-v_{2}, \\
\left\langle-l^{\prime}, u\right\rangle & \leq \max \left\{-\mu_{1},-\mu_{2}\right\}-\max \left\{-v_{1},-v_{2}\right\}=-\mu_{1}+v_{1}
\end{aligned}
$$

Assume that $v_{2}-v_{1}>\mu_{2}-\mu_{1}$ and that $u \in \mathbb{R}^{n}$ exists with $u \in(\partial g(l)-g(l) l) *(\partial h(l)-h(l) l)$. Then, equations (42) and (43) yield the contradiction

$$
\mu_{1}-v_{1} \leq\left\langle l^{\prime}, u\right\rangle \leq \mu_{2}-v_{2}, \quad \text { i.e. } \quad v_{2}-v_{1} \leq \mu_{2}-\mu_{1}
$$

Now assume that

$$
v_{2}-v_{1} \leq \mu_{2}-\mu_{1}
$$

We will show that

$$
M_{1}=M_{2}
$$

holds for 


$$
M_{1}:=\left(\operatorname{co}\left\{\mu_{1}, \mu_{2}\right\} \cdot l^{\prime}\right) \stackrel{*}{*}\left(\operatorname{co}\left\{v_{1}, v_{2}\right\} \cdot l^{\prime}\right), \quad M_{2}:=\operatorname{co}\left\{\mu_{1}-v_{1}, \mu_{2}-v_{2}\right\} \cdot l^{\prime} .
$$

" $\subset$ ": Let $\eta \in \mathbb{R}^{n}$. Using $\langle l, u\rangle=0$ and the orthonormal basis $\left\{l, l^{\prime}\right\}$, we get $\eta=\langle\eta, l\rangle \cdot l+\left\langle\eta, l^{\prime}\right\rangle \cdot l^{\prime}$ and

$$
\begin{aligned}
\delta^{*}\left(\eta, M_{1}\right) & =\max _{u \in M_{1}}\langle\eta, u\rangle=\max _{u \in M_{1}}\left(\langle\eta, l\rangle \cdot\langle l, u\rangle+\left\langle\eta, l^{\prime}\right\rangle \cdot\left\langle l^{\prime}, u\right\rangle\right) \\
& =\left\langle\eta, l^{\prime}\right\rangle \cdot \delta^{*}\left(l^{\prime}, M_{1}\right) \leq\left\langle\eta, l^{\prime}\right\rangle \cdot\left(\mu_{2}-v_{2}\right) \quad(\text { by }(42)) \\
& =\left\langle\eta, l^{\prime}\right\rangle \cdot \max _{\alpha \in\left[\mu_{1}-v_{1}, \mu_{2}-v_{2}\right]}\left\langle l^{\prime}, \alpha \cdot l^{\prime}\right\rangle \\
& =\max _{\alpha \in\left[\mu_{1}-v_{1}, \mu_{2}-v_{2}\right]}\left(\langle\eta, l\rangle \cdot\left\langle l, \alpha \cdot l^{\prime}\right\rangle+\left\langle\eta, l^{\prime}\right\rangle \cdot\left\langle l^{\prime}, \alpha \cdot l^{\prime}\right\rangle\right) \\
& =\max _{\alpha \in\left[\mu_{1}-v_{1}, \mu_{2}-v_{2}\right]}\left\langle\eta, \alpha \cdot l^{\prime}\right\rangle=\max _{u \in M_{2}}\langle\eta, u\rangle=\delta^{*}\left(\eta, M_{2}\right)
\end{aligned}
$$

which shows that $M_{1} \subset M_{2}$.

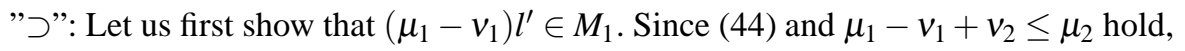

$$
\begin{aligned}
\left(\mu_{1}-v_{1}\right) l^{\prime}+\operatorname{co}\left\{v_{1}, v_{2}\right\} \cdot l^{\prime} & =\left(\mu_{1}-v_{1}\right) l^{\prime}+\operatorname{co}\left\{v_{1} l^{\prime}, v_{2} l^{\prime}\right\} \\
& =\operatorname{co}\left\{\left(\mu_{1}-v_{1}\right) l^{\prime}+v_{1} l^{\prime},\left(\mu_{1}-v_{1}\right) l^{\prime}+v_{2} l^{\prime}\right\} \\
& =\operatorname{co}\left\{\mu_{1} l^{\prime},\left(\mu_{1}-v_{1}+v_{2}\right) l^{\prime}\right\}=\operatorname{co}\left\{\mu_{1},\left(\mu_{1}-v_{1}+v_{2}\right)\right\} \cdot l^{\prime} \\
& \subset \operatorname{co}\left\{\mu_{1}, \mu_{2}\right\} \cdot l^{\prime}
\end{aligned}
$$

Hence, the first endpoint of the line segment $M_{2}$ lies in $M_{1}$ :

$$
\left(\mu_{1}-v_{1}\right) l^{\prime} \in\left(\operatorname{co}\left\{\mu_{1}, \mu_{2}\right\} \cdot l^{\prime}\right) *\left(\operatorname{co}\left\{v_{1}, v_{2}\right\} \cdot l^{\prime}\right)=M_{1}
$$

Now, we proceed similarly with the second endpoint $\left(\mu_{2}-v_{2}\right) l^{\prime}$. Since (44) and $\mu_{2}-v_{2}+v_{1} \geq \mu_{1}$ is valid,

$$
\begin{aligned}
\left(\mu_{2}-v_{2}\right) l^{\prime}+\operatorname{co}\left\{v_{1}, v_{2}\right\} \cdot l^{\prime} & =\left(\mu_{2}-v_{2}\right) l^{\prime}+\operatorname{co}\left\{v_{1} l^{\prime}, v_{2} l^{\prime}\right\} \\
& =\operatorname{co}\left\{\left(\mu_{2}-v_{2}\right) l^{\prime}+v_{1} l^{\prime},\left(\mu_{2}-v_{2}\right) l^{\prime}+v_{2} l^{\prime}\right\} \\
& =\operatorname{co}\left\{\left(\mu_{2}-v_{2}+v_{1}\right) l^{\prime}, \mu_{2} l^{\prime}\right\}=\operatorname{co}\left\{\left(\mu_{2}-v_{2}+v_{1}\right), \mu_{2}\right\} \cdot l^{\prime} \\
& \subset \operatorname{co}\left\{\mu_{1}, \mu_{2}\right\} \cdot l^{\prime}
\end{aligned}
$$

An immediate consequence is that the second endpoint of $M_{2}$ also lies in $M_{1}$ :

$$
\left(\mu_{2}-v_{2}\right) l^{\prime} \in\left(\operatorname{co}\left\{\mu_{1}, \mu_{2}\right\} \cdot l^{\prime}\right) *\left(\operatorname{co}\left\{v_{1}, v_{2}\right\} \cdot l^{\prime}\right)=M_{1}
$$

Since $M_{1}$ is convex, it follows that

$$
M_{2}=\operatorname{co}\left\{\left(\mu_{1}-v_{1}\right) l^{\prime},\left(\mu_{2}-v_{2}\right) l^{\prime}\right\} \subset M_{1} .
$$

This equality for both sets is used to reformulate the geometric difference:

$$
\begin{aligned}
\partial g(l) * \partial h(l) & =(\partial g(l)-g(l) l) *(\partial h(l)-h(l) l)+f(l) l \\
& =\left(\operatorname{co}\left\{\mu_{1}, \mu_{2}\right\} \cdot l^{\prime}\right) *\left(\operatorname{co}\left\{v_{1}, v_{2}\right\} \cdot l^{\prime}\right)+f(l) l=\operatorname{co}\left\{\mu_{1}-v_{1}, \mu_{2}-v_{2}\right\} \cdot l^{\prime}+f(l) l \\
& =\operatorname{co}\left\{\left(\mu_{1}-v_{1}\right) l^{\prime}+f(l) l,\left(\mu_{2}-v_{2}\right) l^{\prime}+f(l) l\right\}
\end{aligned}
$$

Let us now calculate both endpoints of the line segment using (41):

$$
\begin{aligned}
\left(\mu_{1}-v_{1}\right) l^{\prime}+f(l) l= & \left(\left\langle d_{g}\left(l ;-l^{\prime}\right), l^{\prime}\right\rangle-\left\langle d_{h}\left(l ;-l^{\prime}\right), l^{\prime}\right\rangle\right) \cdot l^{\prime}+g(l) l-h(l) l \\
= & \left(\left\langle d_{g}\left(l ;-l^{\prime}\right), l^{\prime}\right\rangle \cdot l^{\prime}+\left\langle d_{g}\left(l ;-l^{\prime}\right), l\right\rangle \cdot l\right) \\
& -\left(\left\langle d_{h}\left(l ;-l^{\prime}\right), l^{\prime}\right\rangle \cdot l^{\prime}+\left\langle d_{h}\left(l ;-l^{\prime}\right), l\right\rangle \cdot l\right) \\
= & d_{g}\left(l ;-l^{\prime}\right)-d_{h}\left(l ;-l^{\prime}\right), \\
\left(\mu_{2}-v_{2}\right) l^{\prime}+f(l) l= & \left(\left\langle d_{g}\left(l ; l^{\prime}\right), l^{\prime}\right\rangle-\left\langle d_{h}\left(l ; l^{\prime}\right), l^{\prime}\right\rangle\right) \cdot l^{\prime}+g(l) l-h(l) l \\
= & \left(\left\langle d_{g}\left(l ; l^{\prime}\right), l^{\prime}\right\rangle \cdot l^{\prime}+\left\langle d_{g}\left(l ; l^{\prime}\right), l\right\rangle \cdot l\right) \\
& -\left(\left\langle d_{h}\left(l ; l^{\prime}\right), l^{\prime}\right\rangle \cdot l^{\prime}+\left\langle d_{h}\left(l ; l^{\prime}\right), l\right\rangle \cdot l\right) \\
= & d_{g}\left(l ; l^{\prime}\right)-d_{h}\left(l ; l^{\prime}\right)
\end{aligned}
$$


This finally shows that

$$
\partial g(l) * \partial h(l)=\operatorname{co}\left\{d_{g}\left(l ;-l^{\prime}\right)-d_{h}\left(l ;-l^{\prime}\right), d_{g}\left(l ; l^{\prime}\right)-d_{h}\left(l ; l^{\prime}\right)\right\}
$$

The next main theorem shows that the Mordukhovich subdifferential of $f$ at 0 can be represented via visualization parts from (18)-(19) of the directed subdifferential.

Theorem 3.13. Let $f=g-h$, where $g, h: \mathbb{R}^{2} \rightarrow \mathbb{R}$ are sublinear functions, and let $\vec{A}=\vec{\partial} f(0)$ be the directed subdifferential of $f$ at 0 . Then,

$$
\partial_{M} f(0)=P_{2}(\vec{A}) \cup \bigcup_{l \in \mathscr{S}_{1}} Q_{2, l}\left(P_{1}\left(\overrightarrow{A_{1}}(l)\right) \cup \operatorname{bd} N_{1}\left(\overrightarrow{A_{1}}(l)\right)\right),
$$

where bd denotes the boundary of a set in $\mathbb{R}$, and $Q_{2, l}(y)=R_{2, l}^{\top} \pi_{1,2}^{\top}(y)+a_{2}(l)$ is the reprojection as in [3].

Proof. First of all, observe that by Proposition 3.1

$$
\partial_{M} f(0)=\partial_{F} f(0) \cup \bigcup_{l \in \mathscr{S}_{1}}\left(\partial_{F} f(l) \cup \underset{t \downarrow 0}{\operatorname{Limsup}} \partial_{F} f\left(l-t l^{\prime}\right) \cup \underset{t \downarrow 0}{\operatorname{Limsup}} \partial_{F} f\left(l+t l^{\prime}\right)\right),
$$

where $l^{\prime}=R_{2, l}^{\top} e^{1}$. The proof consists of three parts:

Step 1: We will show that the positive part coincides with the Fréchet subdifferential at $x=0$ :

$$
\partial_{F} f(0)=P_{2}(\vec{A})
$$

Step 2: We will conclude that the reprojected positive part is the second term in (46):

$$
\partial_{F} f(l)=\left\{\begin{array}{cc}
Q_{2, l} P_{1}\left(\overrightarrow{A_{1}}(l)\right), & \text { if } P_{1}\left(\overrightarrow{A_{1}}(l)\right) \neq \emptyset, \\
\emptyset, & \text { if } P_{1}\left(\overrightarrow{A_{1}}(l)\right)=\emptyset
\end{array}\right.
$$

Step 3: We will prove the following equality for the reprojected boundary points:

$$
\underset{t \downarrow 0}{\operatorname{Limsup}} \partial_{F} f\left(l-t l^{\prime}\right) \cup \underset{t \downarrow 0}{\operatorname{Limsup}} \partial_{F} f\left(l+t l^{\prime}\right)=Q_{2, l}\left(\operatorname{bd} P_{1}\left(\overrightarrow{A_{1}}(l)\right) \cup \operatorname{bd} N_{1}\left(\overrightarrow{A_{1}}(l)\right)\right)
$$

It is not difficult to see that Steps 1-3 together with (46) yield (45).

Step 1: For the Fréchet subdifferential, Lemma 2.2 yields

$$
\partial_{F} f(0)=\left\{v \mid\langle v, l\rangle \leq f(l) \quad \forall l \in \mathscr{S}_{1}\right\}
$$

This equation can be compared with the definition of the positive part of the directed set:

$$
P_{2}(\vec{A})=\left\{v \in \mathbb{R}^{2} \mid\langle v, l\rangle \leq a_{2}(l) \quad \forall l \in \mathscr{S}_{1}\right\}
$$

Since $a_{2}(l)=g(l)-h(l)=f(l)$, from (50) and (51) we conclude (47).

Step 2: By Lemma 3.12 for all $l \in \mathscr{S}_{1}$ we have

$$
\partial_{F} f(l)=\left\{\begin{array}{cl}
\operatorname{co}\left\{d_{g}\left(l ;-l^{\prime}\right)-d_{h}\left(l ;-l^{\prime}\right), d_{g}\left(l ; l^{\prime}\right)-d_{h}\left(l ; l^{\prime}\right)\right\}, & \text { if case } 1 \text { of Lemma } 3.12 \text { holds } \\
\emptyset, & \text { if the opposite inequality holds }
\end{array}\right.
$$

where the notation (5) is again used.

At the same time, Lemma 3.10 yields for every $l \in \mathscr{S}_{1}$

$$
\begin{aligned}
& P_{1}\left(\overrightarrow{A_{1}}(l)\right)=P_{1}\left(\overrightarrow{\left.\left\langle d_{g}\left(l ;-l^{\prime}\right)-d_{h}\left(l ;-l^{\prime}\right), l^{\prime}\right\rangle,\left\langle d_{g}\left(l ; l^{\prime}\right)-d_{h}\left(l ; l^{\prime}\right), l^{\prime}\right\rangle\right]}\right) \\
& =\left\{\begin{array}{cl}
{\left[\left\langle d_{g}\left(l ;-l^{\prime}\right)-d_{h}\left(l ;-l^{\prime}\right), l^{\prime}\right\rangle,\left\langle d_{g}\left(l ; l^{\prime}\right)-d_{h}\left(l ; l^{\prime}\right), l^{\prime}\right\rangle\right],} & \text { if case } 1 \text { of Lemma } 3.12 \text { holds, } \\
\emptyset, & \text { if case } 2 \text { of Lemma } 3.12 \text { holds. }
\end{array}\right.
\end{aligned}
$$

Observe that 


$$
\begin{aligned}
& Q_{2, l}\left(\left\langle d_{g}\left(l ; \pm l^{\prime}\right)-d_{h}\left(l ; \pm l^{\prime}\right), l^{\prime}\right\rangle\right) \\
= & R_{2, l}^{\top} \pi_{1,2}^{\top}\left(\left\langle d_{g}\left(l ; \pm l^{\prime}\right)-d_{h}\left(l ; \pm l^{\prime}\right), l^{\prime}\right\rangle\right)+a_{2}(l) l \\
= & R_{2, l}^{\top}\left(\left\langle d_{g}\left(l ; \pm l^{\prime}\right)-d_{h}\left(l ; \pm l^{\prime}\right), l^{\prime}\right\rangle\right) e^{1}+f(l) l \\
= & \left\langle d_{g}\left(l ; \pm l^{\prime}\right)-d_{h}\left(l ; \pm l^{\prime}\right), l^{\prime}\right\rangle \cdot l^{\prime}+(g(l)-h(l)) l \\
= & \left\langle d_{g}\left(l ; \pm l^{\prime}\right), l^{\prime}\right\rangle \cdot l^{\prime}+g(l) l-\left\langle d_{h}\left(l ; \pm l^{\prime}\right), l^{\prime}\right\rangle \cdot l^{\prime}-h(l) l \\
= & \left\langle d_{g}\left(l ; \pm l^{\prime}\right), l^{\prime}\right\rangle \cdot l^{\prime}+\left\langle d_{g}\left(l ; \pm l^{\prime}\right), l\right\rangle \cdot l \\
& -\left\langle d_{h}\left(l ; \pm l^{\prime}\right), l^{\prime}\right\rangle \cdot l^{\prime}-\left\langle d_{h}\left(l ; \pm l^{\prime}\right), l\right\rangle \cdot l \\
= & d_{g}\left(l ; \pm l^{\prime}\right)-d_{h}\left(l ; \pm l^{\prime}\right),
\end{aligned}
$$

and hence

$$
\begin{aligned}
& Q_{2, l}\left\{\left[\left\langle d_{g}\left(l ;-l^{\prime}\right)-d_{h}\left(l ;-l^{\prime}\right), l^{\prime}\right\rangle,\left\langle d_{g}\left(l ; l^{\prime}\right)-d_{h}\left(l ; l^{\prime}\right), l^{\prime}\right\rangle\right]\right\} \\
= & \operatorname{co}\left\{d_{g}\left(l ;-l^{\prime}\right)-d_{h}\left(l ;-l^{\prime}\right), d_{g}\left(l ; l^{\prime}\right)-d_{h}\left(l ; l^{\prime}\right)\right\} .
\end{aligned}
$$

From (52), (53) and (55) the equation (48) follows.

Step 3: By Lemma 3.6

$$
\underset{t \downarrow 0}{\operatorname{Limsup}} \partial_{F} f\left(l \pm t l^{\prime}\right)=\left\{d_{g}\left(l ; \pm l^{\prime}\right)-d_{h}\left(l ; \pm l^{\prime}\right)\right\}
$$

since $l \perp l^{\prime}$. An immediate consequence of (53) and (54) is

$$
Q_{2, l}\left(\operatorname{bd} P_{1}\left(\overrightarrow{A_{1}}(l)\right)\right)=\left\{\begin{array}{cl}
\left\{d_{g}\left(l ;-l^{\prime}\right)-d_{h}\left(l ;-l^{\prime}\right), d_{g}\left(l ; l^{\prime}\right)-d_{h}\left(l ; l^{\prime}\right)\right\}, & \text { if } P_{1}\left(\overrightarrow{A_{1}}(l)\right) \neq \emptyset, \\
\emptyset, & \text { if } P_{1}\left(\overrightarrow{A_{1}}(l)\right)=\emptyset .
\end{array}\right.
$$

Since $N_{1}\left(\overrightarrow{A_{1}}(l)\right)=\ominus P_{1}\left(-\overrightarrow{A_{1}}(l)\right)$, the expression for $Q_{2, l}\left(\right.$ bd $\left.N_{1}\left(\overrightarrow{A_{1}}(l)\right)\right)$ can be obtained analogously:

$$
Q_{2, l}\left(\operatorname{bd} N_{1}\left(\overrightarrow{A_{1}}(l)\right)\right)=\left\{\begin{array}{cc}
\left\{d_{g}\left(l ;-l^{\prime}\right)-d_{h}\left(l ;-l^{\prime}\right), d_{g}\left(l ; l^{\prime}\right)-d_{h}\left(l ; l^{\prime}\right)\right\}, & \text { if } N_{1}\left(\overrightarrow{A_{1}}(l)\right) \neq \emptyset, \\
\emptyset, & \text { if } N_{1}\left(\overrightarrow{A_{1}}(l)\right)=\emptyset
\end{array}\right.
$$

There are three possible cases (see [3, Proposition 3.4]): either one of the sets $\left.P_{1}\left(\overrightarrow{A_{1}}(l)\right)\right)$ or $\left.N_{1}\left(\overrightarrow{A_{1}}(l)\right)\right)$ is empty or both are singletons and $\left.\left.P_{1}\left(\overrightarrow{A_{1}}(l)\right)\right)=N_{1}\left(\overrightarrow{A_{1}}(l)\right)\right)$. Together with (57) and (58) this yields

$$
Q_{2, l}\left(\operatorname{bd} P_{1}\left(\overrightarrow{A_{1}}(l)\right) \cup \operatorname{bd} N_{1}\left(\overrightarrow{A_{1}}(l)\right)\right)=\left\{d_{g}\left(l ;-l^{\prime}\right)-d_{h}\left(l ;-l^{\prime}\right), d_{g}\left(l ; l^{\prime}\right)-d_{h}\left(l ; l^{\prime}\right)\right\}
$$

Now, (56) and (59) yield (49).

The Mordukhovich superdifferential and symmetric subdifferential of $f$ at 0 is represented via the directed subdifferential in the following theorem. Besides isolated points from the reprojected lower dimensional positive part of the directed subdifferential, the Mordukhovich superdifferential forms the negative two- and one-dimensional part in the visualization of the directed subdifferential. The positive parts are reflected by the Mordukhovich subdifferential (see Theorem 3.13) so that the Mordukhovich symmetric subdifferential form the complete visualization of the directed subdifferential for DS functions.

Theorem 3.14. Let $f=g-h$, where $g, h: \mathbb{R}^{2} \rightarrow \mathbb{R}$ are sublinear functions, and let $\vec{A}=\vec{\partial} f(0)$ be the directed subdifferential of $f$ at 0 . Then,

$$
\begin{aligned}
& \partial_{M}^{+} f(0)=N_{2}(\vec{A}) \cup \bigcup_{l \in \mathscr{S}_{1}} Q_{2, l}\left(N_{1}\left(\overrightarrow{A_{1}}(l)\right) \cup \operatorname{bd} P_{1}\left(\overrightarrow{A_{1}}(l)\right)\right), \\
& \partial_{M}^{0} f(0)=V_{2}(\vec{\partial} f(0)) .
\end{aligned}
$$

Proof. Apply Theorem 3.13 to $-f=h-g$ and use [3, Proposition 3.8]:

$$
\begin{aligned}
\vec{\partial}(-f)(0) & =-\vec{\partial} f(0), & \ominus P_{2}(-\vec{A}) & =N_{2}(\vec{A}), \\
\ominus P_{1}\left(-\overrightarrow{A_{1}}(l)\right) & =N_{1}\left(\overrightarrow{A_{1}}(l)\right), & \ominus N_{1}\left(-\overrightarrow{A_{1}}(l)\right) & =P_{1}\left(\overrightarrow{A_{1}}(l)\right)
\end{aligned}
$$


Together with (15), equation (60) follows immediately.

Since

$$
V_{1}\left(\overrightarrow{A_{1}}(l)\right)=P_{1}\left(\overrightarrow{A_{1}}(l)\right) \cup N_{1}\left(\overrightarrow{A_{1}}(l)\right), \quad M_{2}(\vec{A}) \subset Q_{2, l} V_{1}\left(\overrightarrow{A_{1}}(l)\right)
$$

and (18) hold, the second equation (61) follows easily.

Applying the previous Theorems 3.11,3.13 and 3.14 to the directional derivative generalizes these results to the class of general DC functions (which are not necessarily positively homogeneous).

As a starting point, we will demonstrate that the directed subdifferential of the function at $x$ coincides with the one of its directional derivative evaluated at direction $l=0$.

Proposition 3.15. Let $f=g-h$ with $g, h: \mathbb{R}^{n} \rightarrow \mathbb{R}$ be convex functions. Then,

$$
\vec{\partial}\left[f^{\prime}(x ; \cdot)\right](0)=\vec{\partial} f(x) .
$$

Proof. By Lemma 3.3, the convex subdifferential of $g^{\prime}(x ;)$ in 0 coincides with the one of $g(x)$ :

$$
\partial\left[g^{\prime}(x ; \cdot)\right](0)=Y(0, \partial g(x))=\partial g(x)
$$

The same is true for the convex function $h$ such that

$$
\vec{\partial}\left[f^{\prime}(x ; \cdot)\right](0)=J_{n}\left(\partial\left[g^{\prime}(x ; \cdot)\right](0)\right)-J_{n}\left(\partial\left[h^{\prime}(x ; \cdot)\right](0)\right)=J_{n}(\partial g(x))-J_{n}(\partial h(x))=\vec{\partial} f(x) .
$$

Since the Mordukhovich subdifferential of the directional derivative may differ from the one for the function itself (see Example 4.3) in contrary to the directed subdifferential, the following results for the Mordukhovich subdifferentials have to be formulated with the directional derivative. The next theorem yields the connection between outer limits of Fréchet subdifferentials and the directed subdifferential.

Theorem 3.16. Let $g, h: \mathbb{R}^{2} \rightarrow \mathbb{R}$ be convex functions, and let $f=g-h$. Then the directed subdifferential $\vec{A}=$ $\left(\overrightarrow{A_{1}}(l), a_{2}(l)\right)_{l \in \mathscr{S}_{1}}$ of $f$ at $x$ can be constructed via limits of Fréchet normals as follows: for every $l \in \mathscr{S}_{1}$ let

$$
f_{2}(l):=f^{\prime}(x ; l), \quad \overrightarrow{F_{1}}(l):=\overrightarrow{\left[\pi_{1,2} R_{2, l}\left(D^{-}(l)-f^{\prime}(x ; l) l\right), \pi_{1,2} R_{2, l}\left(D^{+}(l)-f^{\prime}(x ; l) l\right)\right]},
$$

where

$$
D^{-}(l):=\underset{t \downarrow 0}{\operatorname{Limsup}} \partial_{F} f^{\prime}(x ; \cdot)\left(l-t l^{\prime}\right), \quad D^{+}(l):=\underset{t \downarrow 0}{\operatorname{Limsup}} \partial_{F} f^{\prime}(x ; \cdot)\left(l+t l^{\prime}\right),
$$

and $l^{\prime}:=R_{2, l}^{\top} e^{1}$.

Then, $\vec{F}=\left(\overrightarrow{F_{1}}(l), f_{2}(l)\right)_{l \in \mathscr{S}_{1}}$ coincides with $\vec{A}=\vec{\partial} f(x)$.

Proof. Applying [10, Sect. I.3, Proposition 3.1], the directional derivative

$$
f^{\prime}(x ; l)=g^{\prime}(x ; l)-h^{\prime}(x ; l)
$$

is a DS representation. Hence, Proposition 3.15 and Theorem 3.11 can be applied.

The next theorem for DC functions, in which we can drop the assumption of positive homogeneity, could be seen as the non-convex counterpart of the following result for locally Lipschitz and directionally differentiable function in [19, Sect. 3] and [8, (35)]:

$$
\partial_{\mathrm{Cl}}\left[f^{\prime}(x ; \cdot)\right](0)=\partial_{M P} f(x),
$$

where $\partial_{M P} f(x)$ is the Michel-Penot subdifferential of $f$ in $x$ (see $[8,21]$ ). In what follows the Mordukhovich symmetric subdifferential for the directional derivative at $x$ in direction 0 coincides with the Rubinov subdifferential at $x$, i.e. its visualized directed subdifferential.

Theorem 3.17. Let $f=g-h$, where $g, h: \mathbb{R}^{2} \rightarrow \mathbb{R}$ are convex functions, and let $\vec{A}=\vec{\partial} f(x)$ be the directed subdifferential of $f$ at $x$. Then, 


$$
\begin{aligned}
& \partial_{M}\left[f^{\prime}(x ; \cdot)\right](0)=P_{2}(\vec{A}) \cup \bigcup_{l \in \mathscr{S}_{1}} Q_{2, l}\left(P_{1}\left(\overrightarrow{A_{1}}(l)\right) \cup \operatorname{bd} N_{1}\left(\overrightarrow{A_{1}}(l)\right)\right), \\
& \partial_{M}^{+}\left[f^{\prime}(x ; \cdot)\right](0)=N_{2}(\vec{A}) \cup \bigcup_{l \in \mathscr{S}_{1}} Q_{2, l}\left(N_{1}\left(\overrightarrow{A_{1}}(l)\right) \cup \operatorname{bd} P_{1}\left(\overrightarrow{A_{1}}(l)\right)\right), \\
& \partial_{M}^{0}\left[f^{\prime}(x ; \cdot)\right](0)=V_{2}(\vec{\partial} f(x)) .
\end{aligned}
$$

Proof. As in the proof of Theorem 3.16 the equality (62) of the directed subdifferential of $f^{\prime}(x ; \cdot)$ in 0 and the one of $f(\cdot)$ in $x$ holds. The claimed equalities are proved by applying Theorems 3.13 and 3.14.

Remark 3.18. All the lemmas starting from Lemma 3.3 could be adapted to the convex (instead of sublinear) situation. For this purpose, the function must be replaced by its directional derivative, which is sublinear with respect to its second argument. E.g., Lemma 3.5 reads for $h$ being only convex:

$$
\underset{t \downarrow 0}{\operatorname{Limsup}} \partial\left[h^{\prime}(x ; \cdot)\right]\left(l+t l^{\prime}\right)=Y\left(l^{\prime}, Y(l, \partial h(x))\right) .
$$

\section{Examples}

For each of the presented examples we will first calculate theoretically the Mordukhovich subdifferential and superdifferential. Their union, the symmetric subdifferential is compared visually with the Rubinov subdifferential in [4].

We will frequently use Lemma 2.3 for evaluating the Fréchet subdifferential which is a basic tool for calculating the Mordukhovich subdifferential with (13). Analogously, we proceed with the Fréchet superdifferential and (14) in the same way to evaluate the Mordukhovich superdifferential.

The first example is governed by a parameter $r$ by which three different cases could be studied: the Mordukhovich subdifferential has nonempty interior $(r=0.5)$, the Mordukhovich superdifferential has nonempty interior $(r=2.0)$ and both have empty interior $(r=1.25)$. This corresponds to nonemptiness of the positive part resp. of the negative part as well as the mere presence of the mixed-type part in the directed subdifferential.

Example 4.1 ([4, Example 5.7]). Let $f=g-h$, where

$$
g(x)=\left|x_{1}\right|+\left|x_{2}\right|, \quad h(x)=r \sqrt{x_{1}^{2}+x_{2}^{2}}=r\|x\|, r>0 .
$$

To evaluate the Mordukhovich lower/upper/symmetric subdifferential of $f$ at zero directly, we first need to calculate the Fréchet subdifferentials of $f$ at zero and in its neighborhood.

A. The Fréchet subdifferential at 0 . Observe that $f$ can be represented as follows:

$$
f(x)=g(x)-r \sqrt{x_{1}^{2}+x_{2}^{2}}=g(x)+\min _{\|w\|=r}\langle w, x\rangle=\min _{\|w\|=r}(g(x)+\langle w, x\rangle) .
$$

Let

$$
\varphi_{w}(x):=\langle w, x\rangle+g(x),
$$

then

$$
f(x)=\min _{\|w\|=r} \varphi_{w}(x) .
$$

Since $f^{\prime}(0 ; l)=f(l)$, the formula $(9)$ for the Fréchet subdifferential holds:

$$
\partial_{F} f(0)=\bigcap_{\|w\|=r}(\partial g(0)+w)
$$

It is not difficult to see that

$$
\partial g(0)=\operatorname{co}\{(1,1),(-1,1),(1,-1),(-1,-1)\}=[-1,1]^{2} .
$$

We are going to show that 


$$
\partial_{F} f(0)= \begin{cases}{[-1+r, 1-r]^{2},} & r \leq 1 \\ \emptyset, & r>1\end{cases}
$$

Let $u \in \partial_{F} f(0)$. For every $w,\|w\|=r$, there exists $v \in[-1,1]^{2}$ by (67) such that the coordinates satisfy

$$
u_{i}=v_{i}+w_{i}, \quad i=1,2 .
$$

This yields $-1+r \leq u_{i} \leq 1-r$, and hence

$$
\partial_{F} f(0) \subset[-1+r, 1-r]^{2}, \quad \text { if } r \leq 1,
$$

and

$$
\partial_{F} f(0)=\emptyset, \quad \text { if } r>1 .
$$

To show the inclusion opposite to (69), consider an arbitrary $u$ such that $-1+r \leq u_{i} \leq 1-r$. For every $w,\|w\|=r$, we set $v:=u-w$. Then $v \in[-1,1]^{2}$ is valid as well as

$$
\partial_{F} f(0) \supset[-1+r, 1-r]^{2} .
$$

Now, (68) follows from (69)-(71).

B. The Fréchet superdifferential at 0 . Observe that

$$
\begin{aligned}
f(x) & =\max _{i=1, \ldots, 4}\left\langle v^{i}, x\right\rangle-\max _{\|w\|=r}\langle w, x\rangle \\
& =\max _{i=1, \ldots, 4}\left\{\left\langle v^{i}, x\right\rangle-\max _{\|w\|=r}\langle w, x\rangle\right\}=-\min _{i=1, \ldots, 4}\left\{\max _{\|w\|=r}\langle w, x\rangle-\left\langle v^{i}, x\right\rangle\right\},
\end{aligned}
$$

where

$$
v^{1}=(1,1), \quad v^{2}=(1,-1), \quad v^{3}=(-1,1), \quad v^{4}=(-1,-1) .
$$

Let

$$
\varphi_{i}(x)=\max _{\|w\|=r}\langle w, x\rangle-\left\langle v^{i}, x\right\rangle
$$

It is not difficult to observe that

$$
\partial \varphi_{i}(x)=\mathbb{B}_{r}(0)-v^{i}=\mathbb{B}_{r}\left(-v^{i}\right),
$$

where $\mathbb{B}_{r}(m)=\{x \mid\|x-m\|=r\}$. Using (10), the Fréchet superdifferential can be calculated as

$$
\partial_{F}^{+} f(x)=\ominus \bigcap_{i=1}^{4} \mathbb{B}_{r}\left(-v^{i}\right)=\bigcap_{i=1}^{4} \mathbb{B}_{r}\left(v^{i}\right) .
$$

C. The Fréchet sub- and superdifferentials around 0 . For every $x \neq 0$ the function $h$ is smooth, hence

$$
\partial_{F} f(x)=\partial f(x)=\partial g(x)-h^{\prime}(x)=\partial g(x)-r \frac{x}{\|x\|} \quad \forall x \neq 0 .
$$

For the Fréchet superdifferential in all points $x \neq 0$ we have

$$
\partial_{F}^{+} f(x)= \begin{cases}g^{\prime}(x)-r \frac{x}{\|x\|}, & \text { if } g \text { is differentiable at } x, \\ \emptyset, & \text { otherwise, since } g \text { is not Fréchet superdifferentiable } \\ & \text { due to [18, Proposition 1.3] }\end{cases}
$$

Observe that for $x \neq 0$, the subdifferential of $g$ is given by

$$
\partial g(x)= \begin{cases}\left\{\left(\operatorname{sgn}\left(x_{1}\right), \operatorname{sgn}\left(x_{2}\right)\right)\right\}, & x_{1} \neq 0, x_{2} \neq 0 \\ \operatorname{co}\{(1,1),(1,-1)\}, & x_{1}>0, x_{2}=0 \\ \operatorname{co}\{(1,1),(-1,1)\}, & x_{1}=0, x_{2}>0 \\ \operatorname{co}\{(-1,-1),(-1,1)\}, & x_{1}<0, x_{2}=0 \\ \operatorname{co}\{(-1,-1),(1,-1)\}, & x_{1}=0, x_{2}<0\end{cases}
$$


Therefore,

$$
\partial_{F} f(x)= \begin{cases}\left\{\left(\operatorname{sgn}\left(x_{1}\right), \operatorname{sgn}\left(x_{2}\right)\right)-r \frac{x}{\|x\|}\right\}, & x_{1} \neq 0, x_{2} \neq 0, \\ \operatorname{co}\{(1,1),(1,-1)\}-\left\{r \frac{x}{\|x\|}\right\}, & x_{1}>0, x_{2}=0, \\ \operatorname{co}\{(1,1),(-1,1)\}-\left\{r \frac{x}{\|x\|}\right\}, & x_{1}=0, x_{2}>0, \\ \operatorname{co}\{(-1,-1),(-1,1)\}-\left\{r \frac{x}{\|x\|}\right\}, & x_{1}<0, x_{2}=0, \\ \operatorname{co}\{(-1,-1),(1,-1)\}-\left\{r \frac{x}{\|x\|}\right\}, & x_{1}=0, x_{2}<0,\end{cases}
$$

and

$$
\partial_{F}^{+} f(x)= \begin{cases}\left\{\left(\operatorname{sgn}\left(x_{1}\right), \operatorname{sgn}\left(x_{2}\right)\right)-r \frac{x}{\|x\|}\right\}, & x_{1} \neq 0, x_{2} \neq 0, \\ \emptyset, & x_{1} x_{2}=0, x \neq 0 .\end{cases}
$$

It is not difficult to observe that for every $l \in S_{1}$ with $l_{1} l_{2} \neq 0$, we have

$$
\underset{t \downarrow 0}{\operatorname{Limsup}} \partial_{F} f\left(l+t l^{\prime}\right)=\partial_{F} f(l), \quad \operatorname{Limsup}_{t \downarrow 0} \partial_{F}^{+} f\left(l+t l^{\prime}\right)=\partial_{F}^{+} f(l) .
$$

by applying (73) and (74). For $l=(1,0)$ and $l^{\prime}=(0,1)$

$$
\begin{aligned}
\underset{t \downarrow 0}{\operatorname{Limsup}} \partial_{F} f\left(l+t l^{\prime}\right)=\underset{t \downarrow 0}{\operatorname{Limsup}} \partial_{F}^{+} f\left(l+t l^{\prime}\right) & =\underset{t \downarrow 0}{\operatorname{Limsup}}\left\{(1,1)-r \frac{l+t l^{\prime}}{\left\|l+t l^{\prime}\right\|}\right\} \\
& =\underset{t \downarrow 0}{\operatorname{Limsup}}\left\{\left(1-\frac{r}{\left\|l+t l^{\prime}\right\|}, 1-\frac{r t}{\left\|l+t l^{\prime}\right\|}\right)\right\} \\
& =\{(1-r, 1)\} .
\end{aligned}
$$

The corresponding outer limits for the remaining directions can be evaluated analogously. We have

$$
\underset{t \downarrow 0}{\operatorname{Limsup}} \partial_{F} f\left(l+t l^{\prime}\right)=\underset{t \downarrow 0}{\operatorname{Limsup}} \partial_{F}^{+} f\left(l+t l^{\prime}\right)=\left\{\begin{aligned}
\{(1-r, 1)\}, & l=(1,0), l^{\prime}=(0,1), \\
\{(1-r,-1)\}, & l=(1,0), l^{\prime}=(0,1), \\
\{(1,1-r)\}, & l=(0,1), l^{\prime}=(1,0), \\
\{(-1,1-r)\}, & l=(0,1), l^{\prime}=(-1,0), \\
\{(-1+r, 1)\}, & l=(-1,0), l^{\prime}=(1,0), \\
\{(-1+r,-1)\}, & l=(-1,0), l^{\prime}=(-1,0), \\
\{(1,-1+r)\}, & l=(0,-1), l^{\prime}=(1,0), \\
\{(-1,-1+r)\}, & l=(0,-1), l^{\prime}=(-1,0) .
\end{aligned}\right.
$$

D. The Mordukhovich subdifferentials at 0 . To finish the evaluation of the Mordukhovich subdifferential, we use Proposition 3.1.

From (68), (73), (75) and (76) the Mordukhovich subdifferential is given by

$$
\begin{aligned}
\partial_{M} f(0)= & \partial_{F} f(0) \cup \operatorname{Limsup}_{\substack{x \rightarrow 0, x \neq 0}} \partial_{F} f(x) \\
= & \left\{u \mid-1+r \leq u_{i} \leq 1-r, i=1,2\right\} \\
& \cup\left[\{(1,1)\}+\left\{w \mid\|w\|=r, w_{1} \leq 0, w_{2} \leq 0\right\}\right] \\
& \cup\left[\{(-1,1)\}+\left\{w \mid\|w\|=r, w_{1} \geq 0, w_{2} \leq 0\right\}\right] \\
& \cup\left[\{(1,-1)\}+\left\{w \mid\|w\|=r, w_{1} \leq 0, w_{2} \geq 0\right\}\right] \\
& \cup\left[\{(-1,-1)\}+\left\{w \mid\|w\|=r, w_{1} \geq 0, w_{2} \geq 0\right\}\right] \\
& \cup \operatorname{co}\{(1-r, 1),(1-r,-1)\} \\
& \cup \operatorname{co}\{(-1,1-r),(1,1-r)\} \\
& \cup \operatorname{co}\{(-1+r,-1),(-1+r, 1)\} \\
& \cup \operatorname{co}\{(-1,-1+r),(1,-1+r)\} .
\end{aligned}
$$

Analogously, from (72) and (74)-(76) 


$$
\begin{aligned}
\partial_{M}^{+} f(0)= & \partial_{F}^{+} f(0) \cup \underset{\substack{x \rightarrow 0, x \neq 0}}{\operatorname{Limsup}} \partial_{F}^{+} f(x) \\
= & \bigcap_{\mu=1}^{4} \mathbb{B}_{r}\left(v^{\mu}\right) \\
& \cup\left[\{(1,1)\}+\left\{w \mid\|w\|=r, w_{1} \leq 0, w_{2} \leq 0\right\}\right] \\
& \cup\left[\{(-1,1)\}+\left\{w \mid\|w\|=r, w_{1} \geq 0, w_{2} \leq 0\right\}\right] \\
& \cup\left[\{(1,-1)\}+\left\{w \mid\|w\|=r, w_{1} \leq 0, w_{2} \geq 0\right\}\right] \\
& \cup\left[\{(-1,-1)\}+\left\{w \mid\|w\|=r, w_{1} \geq 0, w_{2} \geq 0\right\}\right] .
\end{aligned}
$$

The Mordukhovich subdifferentials of $f$ at 0 for the values of $r=0.5,1.25$ and 2.0 are plotted in Figs. 1-3.

The corresponding series for the visualization of the directed subdifferentials with the same values of $r$ are plotted in Fig. 4, see also [4, Example 5.7] for further explanations. The plots coincide with the pictures of the Mordukhovich symmetric subdifferentials. Since the subdifferentials of the convex functions $g$ and $h$ are known, the Rubinov subdifferential could be easily calculated as the visualization of the difference of these embedded convex sets.

The arrows in Fig. 4 indicate outer normals to the directed "supporting faces". They also form the parametrizing directions in (16) for the directed subdifferential. The positive part in the left picture of Fig. 4 is a convex set. It is coloured in gray and only outer normals are attached to its boundary. The other non-convex part belongs to the mixed-type part. Similarly for the right picture in Fig. 4. The gray convex subset is the negative part and has only inner normals attached to its boundary. The positive and negative part in the middle picture are empty and the Rubinov subdifferential consists only of the mixed-type part. Note that the unique "supporting points" belong both to the Mordukhovich subdifferential and superdifferential due to Theorems 3.13 and 3.14, since for such a point the lower dimensional positive and negative parts coincide with the point itself.

Example 4.2 ([23, Example 2.49]). Let

$$
f\left(x_{1}, x_{2}\right):=|| x_{1}\left|+x_{2}\right| .
$$

Straightforward computation of the Mordukhovich subdifferentials of $f$ (see [23], Example 2.49) gives

$$
\begin{aligned}
& \partial_{M} f(0,0)=\operatorname{co}\{(0,0),(1,1),(-1,1)\} \cup \operatorname{co}\{(0,0),(-1,-1)\} \cup \operatorname{co}\{(0,0),(1,-1)\}, \\
& \partial_{M}^{+} f(0,0)=\operatorname{co}\{(1,-1),(-1,-1)\} \cup\{(1,1),(-1,1)\},
\end{aligned}
$$

and

$$
\partial_{M}^{0} f(0,0)=\partial_{M} f(0,0) \cup \operatorname{co}\{(1,-1),(-1,-1)\} .
$$

Figs. 5-6 show the comparison between the Mordukhovich lower/upper/symmetric subdifferential with the Rubinov subdifferential. The calculuation of the latter is based on one DC representation of $f$, e.g.

$$
f(x)=\max \left\{2 x_{1}+2 x_{2},-2 x_{1}+2 x_{2}, 0\right\}-\max \left\{x_{1}+x_{2},-x_{1}+x_{2}\right\} .
$$

As in Example 4.1, one can see that the four unique directed "supporting points" $( \pm 1, \pm 1)$ (see Fig. 5) are present both in the Mordukhovich subdifferential and superdifferential.

The only segment $\operatorname{co}\{(-1,-1),(1,-1)\}$ in the Mordukhovich superdifferential may be recognized from the Rubinov subdifferential in Fig. 6 as coming from a negative part of a directed interval, since there are outer normals attached to its ends (see Fig. 6) where the projections are pointing inside the interval, contrary to all the segments in the Mordukhovich subdifferential.

Also the Rubinov subdifferential (the visualization of the directed one in Fig. 6) coincides with the Mordukhovich symmetric subdifferential, according to Theorem 3.14. 


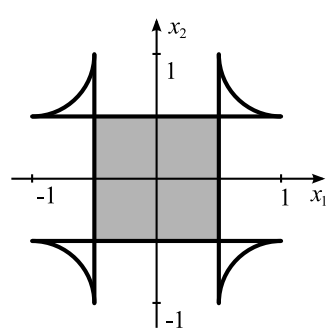

a)

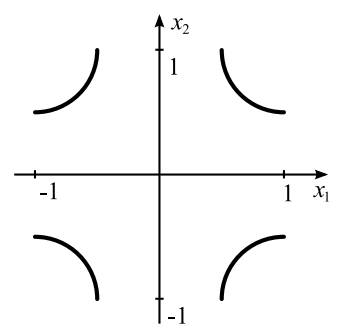

b)

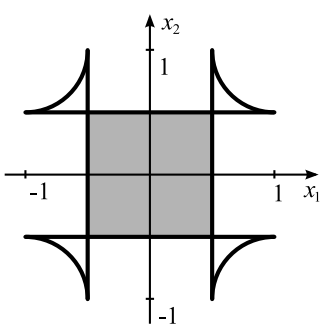

c)

Fig. 1 Mordukhovich subdifferentials of $f$ if $r=0.5$ : a) $\partial_{M} f(0)$; b) $\partial_{M}^{+} f(0)$; c) $\partial_{M}^{0} f(0)$

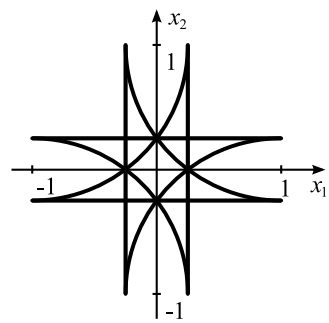

a)

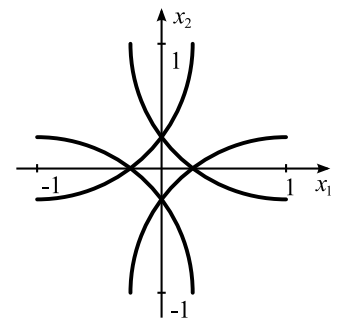

b)

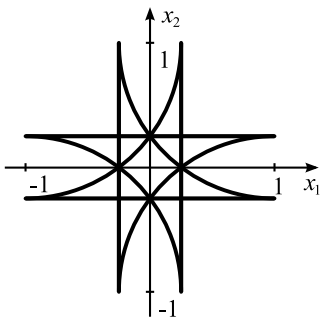

c)

Fig. 2 Mordukhovich subdifferentials of $f$ if $r=1.25:$ a) $\partial_{M} f(0)$; b) $\partial_{M}^{+} f(0)$; c) $\partial_{M}^{0} f(0)$

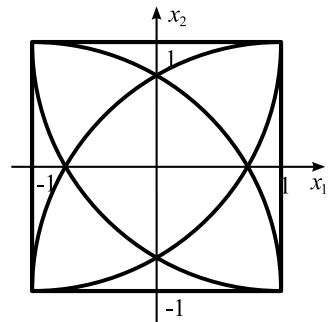

a)

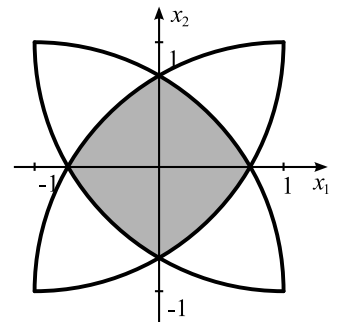

b)

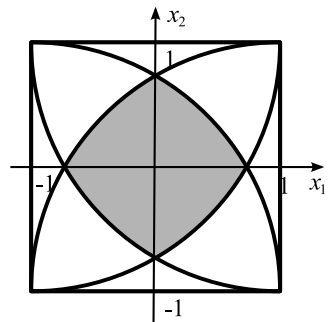

c)

Fig. 3 Mordukhovich subdifferentials of $f$ if $r=2$ : a) $\partial_{M} f(0)$; b) $\partial_{M}^{+} f(0)$; c) $\partial_{M}^{0} f(0)$

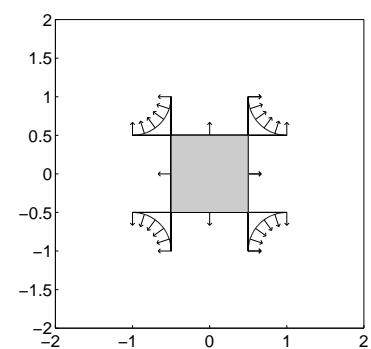

a)

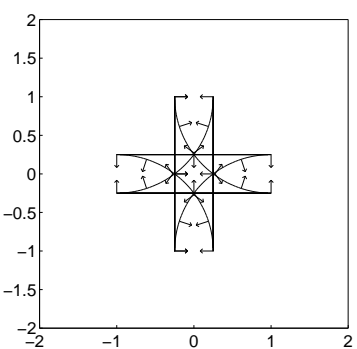

b)

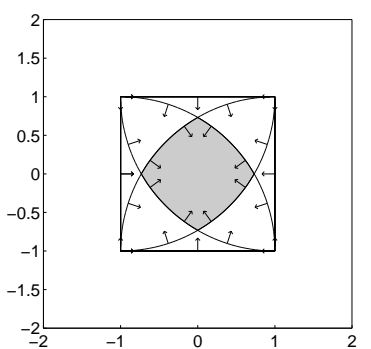

Fig. 4 Visualization of directed subdifferential for Example 4.1 for a) $r=0.5$, b) $r=1.25$, c) $r=2.0$ 


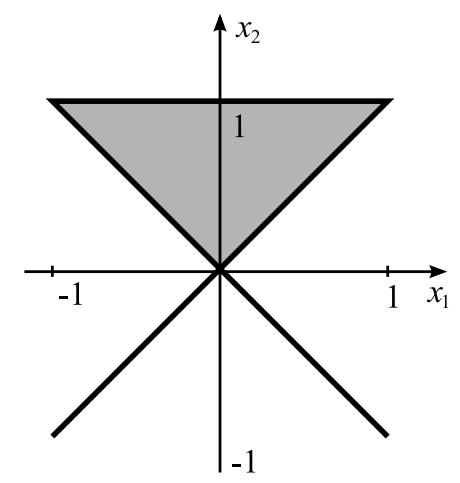

a)

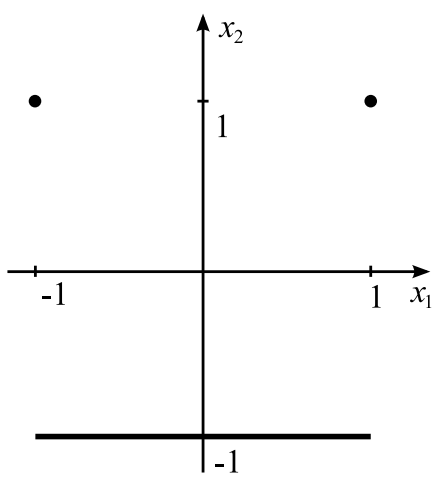

b)

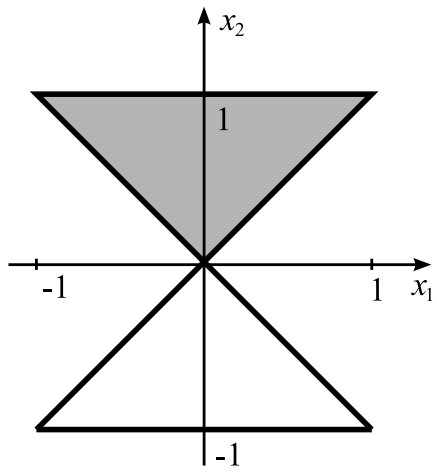

c)

Fig. 5 Mordukhovich subdifferentials for Example 4.2: a) $\partial_{M} f(0)$, b) $\partial_{M}^{+} f(0)$, c) $\partial_{M}^{0} f(0)$

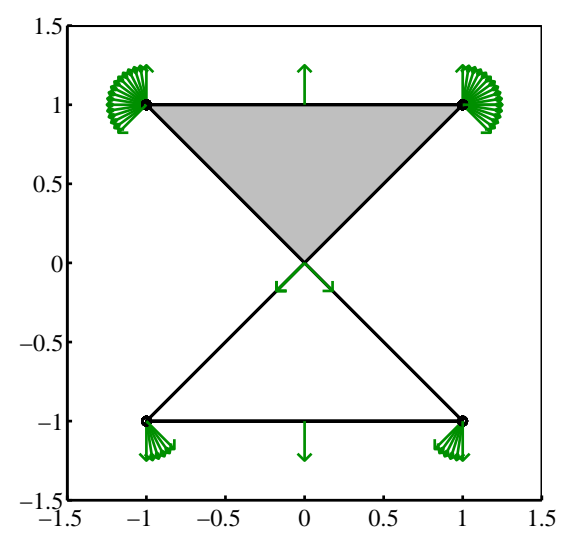

Fig. 6 Visualization of directed subdifferential for Example 4.2

The last example shows the difference between Theorems 3.13/3.14 and 3.17. In this example the function $f$ is $\mathrm{DC}$, but not positive homogeneous. So we cannot expect that we have equality between the Mordukhovich symmetric subdifferential and the Rubinov one (the visualization of the directed subdifferential) as in Theorem 3.14 .

Example 4.3 ([10, Sect. III.4, Example 4.2] and [4, Example 4.7]). Let $f=g-h$, where

$$
g(x)=\max \left\{2 x_{2}, x_{1}^{2}+x_{2}\right\}, \quad h(x)=\max \left\{0, x_{1}^{2}+x_{2}\right\} .
$$

Together with

$$
\varphi_{1}(x)=\max \left\{2 x_{2}, x_{1}^{2}+x_{2}\right\}, \quad \varphi_{2}(x)=\max \left\{0, x_{2}-x_{1}^{2}\right\}
$$

it follows that

$$
\begin{aligned}
f(x) & =\max \left\{2 x_{2}, x_{1}^{2}+x_{2}\right\}+\min \left\{0,-x_{1}^{2}-x_{2}\right\}=\min \left\{\max \left\{2 x_{2}, x_{1}^{2}+x_{2}\right\}, \max \left\{0, x_{2}-x_{1}^{2}\right\}\right\} \\
& =\min \left\{\varphi_{1}(x), \varphi_{2}(x)\right\} .
\end{aligned}
$$


We have

$$
\begin{gathered}
\partial \varphi_{1}(x)= \begin{cases}\{(0,2)\}, & \text { if } x_{2}>x_{1}^{2}, \\
\left\{\left(2 x_{1}, 1\right)\right\}, & \text { if } x_{2}<x_{1}^{2}, \\
\operatorname{co}\left\{(0,2),\left(2 x_{1}, 1\right)\right\}, & \text { if } x_{2}=x_{1}^{2},\end{cases} \\
\partial \varphi_{2}(x)= \begin{cases}\left\{\left(-2 x_{1}, 1\right)\right\}, & \text { if } x_{2}>x_{1}^{2}, \\
\{(0,0)\}, & \text { if } x_{2}<x_{1}^{2}, \\
\operatorname{co}\left\{(0,0),\left(-2 x_{1}, 1\right)\right\}, & \text { if } x_{2}=x_{1}^{2} .\end{cases}
\end{gathered}
$$

It is not difficult to observe that the set of active indices of $f$ in $\mathrm{x}$, i.e.

$$
I_{f}(x)=\left\{i \in\{1,2\} \mid f(x)=\varphi_{i}(x)\right\}= \begin{cases}\{1\}, & \text { if } x_{2}<-x_{1}^{2} \\ \{2\}, & \text { if } x_{2}>-x_{1}^{2} \\ \{1,2\}, & \text { if } x_{2}=-x_{1}^{2}\end{cases}
$$

From Lemma 2.3 follows that

$$
\begin{aligned}
& \partial_{F} f(x)= \begin{cases}\partial \varphi_{1}(x), & \text { if } x_{2}<-x_{1}^{2}, \\
\partial \varphi_{2}(x), & \text { if } x_{2}>-x_{1}^{2}, \\
\partial \varphi_{1}(x) \cap \partial \varphi_{2}(x), & \text { if } x_{2}=-x_{1}^{2}\end{cases} \\
&= \begin{cases}\{(0,0)\}, & \text { if }-x_{1}^{2}<x_{2}<x_{1}^{2}, \\
\left\{\left(-2 x_{1}, 1\right)\right\}, & \text { if } x_{2}>x_{1}^{2}, \\
\left\{\left(2 x_{1}, 1\right)\right\}, & \text { if } x_{2}<-x_{1}^{2}, \\
\operatorname{co}\left\{(0,0),\left(-2 x_{1}, 1\right)\right\}, & \text { if } x_{2}=x_{1}^{2}, x_{1}, x_{2} \neq 0 \\
\emptyset, & \text { if } x_{2}=-x_{1}^{2}, x_{1}, x_{2} \neq 0 \\
\{(0,1)\}, & \text { if } x_{1}=x_{2}=0\end{cases}
\end{aligned}
$$

The evaluation of the outer limit in (13) is straight forward:

$$
\partial_{M} f(0)=\operatorname{Limsup}_{x \rightarrow 0} \partial_{F} f(x)=\operatorname{co}\{(0,0),(0,1)\} .
$$

Since $f$ is Fréchet differentiable, the Rubinov subdifferential yields just the gradient (see [4]):

$$
V_{2}(\vec{\partial} f(0))=\{(0,1)\}
$$

which is a strict subset of the Mordukhovich subdifferential, see Fig. 7.

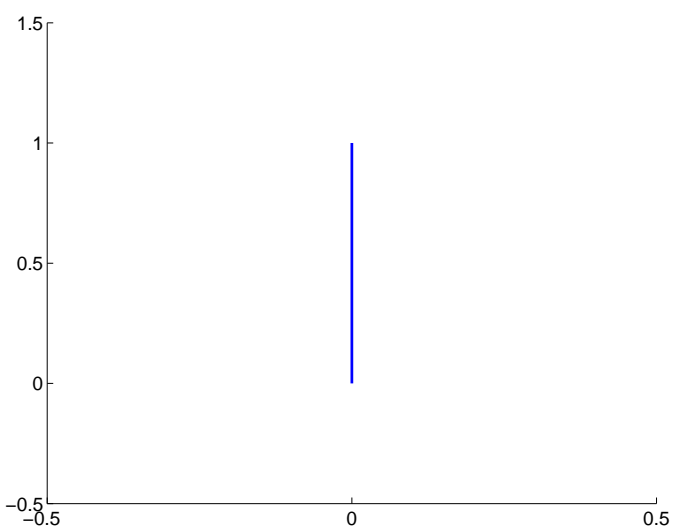

Fig. 7 Mordukhovich and Clarke subdifferential for Example 4.3 
Let us try to apply Theorem 3.17. The formula for the directional derivatives of a DC function is proved in [10, Sect. I.3, Proposition 3.1]:

$$
f^{\prime}(x ; l)=g^{\prime}(x ; l)-h^{\prime}(x ; l)
$$

Since the directional derivatives of $g$ and $h$ involve a maximum, we set

$$
\begin{array}{ll}
g_{1}(x)=2 x_{2}, & g_{2}(x)=x_{1}^{2}+x_{2}, \\
h_{1}(x)=0, & h_{2}(x)=g_{2}(x)
\end{array}
$$

and apply [10, Sect. I.3, Proposition 3.1]:

$$
\begin{array}{ll}
g^{\prime}(x ; l)=\max _{i \in I_{g}(x)} g_{i}^{\prime}(x ; l), & I_{g}(x)=\left\{i \in\{1,2\} \mid g(x)=g_{i}(x)\right\} \\
h^{\prime}(x ; l)=\max _{i \in I_{h}(x)} h_{i}^{\prime}(x ; l), & I_{h}(x)=\left\{i \in\{1,2\} \mid h(x)=h_{i}(x)\right\} .
\end{array}
$$

Now,

$$
\begin{array}{r}
g^{\prime}(x ; l)= \begin{cases}g_{1}^{\prime}(x ; l)=\nabla g_{1}(x) l=(0,2) \cdot\left(\begin{array}{l}
l_{1} \\
l_{2}
\end{array}\right)=2 l_{2}, & \text { if } x_{2}>x_{1}^{2}, \\
g_{2}^{\prime}(x ; l)=\nabla g_{2}(x) l=\left(2 x_{1}, 1\right) \cdot\left(\begin{array}{l}
l_{1} \\
l_{2}
\end{array}\right)=2 x_{1} l_{1}+l_{2}, & \text { if } x_{2}<x_{1}^{2}, \\
\max \left\{g_{1}^{\prime}(x ; l), g_{2}^{\prime}(x ; l)\right\}=\max \left\{2 l_{2}, 2 x_{1} l_{1}+l_{2}\right\}, & \text { if } x_{2}=x_{1}^{2},\end{cases} \\
h^{\prime}(x ; l)= \begin{cases}h_{1}^{\prime}(x ; l)=\nabla h_{1}(x) l=(0,0) \cdot\left(\begin{array}{l}
l_{1} \\
l_{2}
\end{array}\right)=0, & \text { if } x_{2}<-x_{1}^{2}, \\
h_{2}^{\prime}(x ; l)=\nabla h_{2}(x) l=\left(2 x_{1}, 1\right) \cdot\left(\begin{array}{l}
l_{1} \\
l_{2}
\end{array}\right)=2 x_{1} l_{1}+l_{2}, & \text { if } x_{2}>-x_{1}^{2}, \\
\max \left\{h_{1}^{\prime}(x ; l), h_{2}^{\prime}(x ; l)\right\}=\max \left\{0,2 x_{1} l_{1}+l_{2}\right\}, & \text { if } x_{2}=-x_{1}^{2} .\end{cases}
\end{array}
$$

Since we fix $x=0$, we have $x_{2}=-x_{1}^{2}$ and $x_{2}=x_{1}^{2}$ and hence,

$$
\begin{aligned}
f^{\prime}(0 ; l) & =\max \left\{2 l_{2}, 2 \cdot 0 \cdot l_{1}+l_{2}\right\}-\max \left\{0,2 \cdot 0 \cdot l_{1}+l_{2}\right\}=\max \left\{2 l_{2}, l_{2}\right\}-\max \left\{0, l_{2}\right\} \\
& =l_{2}+\max \left\{l_{2}, 0\right\}-\max \left\{0, l_{2}\right\}=l_{2}
\end{aligned}
$$

The function $f^{\prime}(0 ; \cdot)$ is continuously differentiable with respect to $l$, hence strict differentiable by [7, Corollary to Proposition 2.2.1]. One can apply [7, Proposition 2.2.4] to show

$$
\partial_{M P} f(0)=\partial_{\mathrm{Cl}}\left[f^{\prime}(0 ; \cdot)\right](0)=\left\{\nabla_{l} f^{\prime}(0 ; \cdot)(0)\right\}=\{(0,1)\}
$$

which coincides with the Rubinov subdifferential.

A similar reasoning shows that the Fréchet subdifferential and superdifferential of the directional derivative coincides with the gradient of $f^{\prime}(0 ; \cdot)$ with respect to $l$ in any direction $\eta$ by [18, Proposition 1.3]. Hence, the Mordukhovich subdifferential and the Mordukhovich superdifferential also equal to the point $(0,1)$ due to (14).

\section{Conclusions}

As we have shown in this paper, the connection between the Mordukhovich subdifferential/superdifferential and the Rubinov subdifferential may provide substantial information related to their computing and in their applications. This relation will be investigated and explored in more details in our further research. Especially, we are currently working on the extension of our results from the class of DC functions to quasidifferentiable functions and on their application to quasidifferential calculus. Another focus of future research will be the case of dimension higher than two.

Acknowledgements The authors would like to thank the referees for their valuable remarks. They also express their gratitude to Boris Mordukhovich for encouraging us to explore the connections between the Rubinov subdifferential and his subdifferential as well as to Vladimir Demyanov who initiated the research to find connections between the quasidifferential and the Mordukhovich subdifferential. This work was partially supported by The Hermann Minkowski Center for Geometry at Tel Aviv University. 


\section{References}

1. Amahroq, T., Penot, J.P., Syam, A.: On the subdifferentiability of the difference of two functions and local minimization. SetValued Anal. 16(4), 413-427 (2008)

2. Baier, R., Farkhi, E.: Differences of Convex Compact Sets in the Space of Directed Sets, Part I: The Space of Directed Sets. Set-Valued Anal. 9(3), 217-245 (2001)

3. Baier, R., Farkhi, E.: Differences of Convex Compact Sets in the Space of Directed Sets, Part II: Visualization of Directed Sets. Set-Valued Anal. 9(3), 247-272 (2001)

4. Baier, R., Farkhi, E.: The directed subdifferential of DC functions. In: A. Leizarowitz, B.S. Mordukhovich, I. Shafrir, A.J. Zaslavski (eds.) Nonlinear Analysis and Optimization II: Optimization. A Conference in Celebration of Alex Ioffe's 70th and Simeon Reich's 60th Birthdays, June 18-24, 2008, Haifa, Israel, AMS Contemporary Mathematics, vol. 513, pp. 27-43. AMS and Bar-Ilan University (2010)

5. Bazaraa, M.S., Goode, J.J., Nashed, M.Z.: On the cones of tangents with applications to mathematical programming. J. Optim. Theory Appl. 13, 389-426 (1974)

6. Borwein, J.M., Strójwas, H.M.: Proximal analysis and boundaries of closed sets in Banach space. I. Theory. Canad. J. Math. 38(2), 431-452 (1986)

7. Clarke, F.H.: Optimization and nonsmooth analysis, Classics in Applied Mathematics, vol. 5. SIAM, Philadelphia, PA (1990) First edition published in John Wiley \& Sons, Inc., New York, 1983

8. Demyanov, V.F., Jeyakumar, V.: Hunting for a smaller convex subdifferential. J. Global Optim. 10(3), 305-326 (1997)

9. Demyanov, V.F., Roshchina, V.A.: Exhausters, optimality conditions and related problems. J. Global Optim. 40(1-3), 71-85 (2008)

10. Demyanov, V.F., Rubinov, A.M.: Constructive nonsmooth analysis, Approximation and Optimization, vol. 7. Verlag Peter Lang, Frankfurt/Main (1995). Russian original "Foundations of nonsmooth analysis, and quasidifferential calculus" published in Nauka, Moscow, 1990

11. Dinh, N., Mordukhovich, B.S., Nghia, T.T.A.: Qualification and optimality conditions for DC programs with infinite constraints. Acta Math. Vietnam. 34(1), 125-155 (2009)

12. Eberhard, A., Nyblom, M.: Jets, generalised convexity, proximal normality and differences of functions. Nonlinear Anal. 34(3), 319-360 (1998)

13. Hadwiger, H.: Minkowskische Addition und Subtraktion beliebiger Punktmengen und die Theoreme von Erhard Schmidt. Mathematische Zeitschrift 53(3), 210-218 (1950)

14. Hiriart-Urruty, J.B.: Generalized differentiability, duality and optimization for problems dealing with differences of convex functions. In: J. Ponstein (ed.) Convexity and Duality in Optimization. Proceedings of the Symposium on Convexity and Duality in Optimization Held at the University of Groningen, The Netherlands June 22, 1984, Lecture Notes in Econom. and Math. Systems, vol. 256, pp. 37-70. Springer, Berlin-Heidelberg-New York-Tokyo (1985)

15. Hiriart-Urruty, J.B., Lemaréchal, C.: Convex Analysis and Minimization Algorithms I. Fundamentals, Grundlehren der mathematischen Wissenschaften, vol. 305. Springer, Berlin-Heidelberg-New York-London-Paris-Tokyo-Hong Kong-BarcelonaBudapest (1993)

16. Hörmander, P.L.: Sur la fonction d'appui des ensembles convexes dans un espace localement convexe. Ark. Mat. 3(12), 181-186 (1954)

17. Ioffe, A.D.: Sous-différentielles approchées de fonctions numériques. C. R. Acad. Sci. Paris Sér. I Math. 292(14), 675-678 (1981)

18. Kruger, A.Y.: On Fréchet subdifferentials. J. Math. Sci. (N. Y.) 116(3), 3325-3358 (2003). Optimization and related topics, 3

19. Kuntz, L., Scholtes, S.: Constraint qualifications in quasidifferentiable optimization. Math. Program. 60(3, Ser. A), 339-347 (1993)

20. Martínez-Legaz, J.E., Seeger, A.: A formula on the approximate subdifferential of the difference of convex functions. Bull. Austral. Math. Soc. 45(1), 37-41 (1992)

21. Michel, P., Penot, J.P.: Calcul sous-différentiel pour des fonctions lipschitziennes et non lipschitziennes. C. R. Acad. Sci. Paris Sér. I Math. 298(12), 269-272 (1984)

22. Mordukhovich, B.S.: Generalized differential calculus for nonsmooth and set-valued mappings. J. Math. Anal. Appl. 183(1), 250-288 (1994)

23. Mordukhovich, B.S.: Variational analysis and generalized differentiation. I Basic theory, Grundlehren der Mathematischen Wissenschaften [Fundamental Principles of Mathematical Sciences], vol. 330. Springer-Verlag, Berlin (2006)

24. Mordukhovich, B.S.: Variational analysis and generalized differentiation. II Applications, Grundlehren der Mathematischen Wissenschaften [Fundamental Principles of Mathematical Sciences], vol. 331. Springer-Verlag, Berlin (2006)

25. Mordukhovich, B.S., Shao, Y.H.: Nonsmooth sequential analysis in Asplund spaces. Trans. Amer. Math. Soc. 348(4), 1235-1280 (1996)

26. Penot, J.P.: Sous-différentiels de fonctions numériques non convexes. C. R. Acad. Sci. Paris Sér. I Math. 278, $1553-1555$ (1974)

27. Penot, J.P.: Calcul sous-différentiel et optimisation. J. Funct. Anal. 27(2), 248-276 (1978)

28. Pontryagin, L.S.: Linear differential games. ii. Sov. Math., Dokl. 8(4), 910-912 (1967)

29. Rockafellar, R.T.: Convex Analysis, Princeton Mathematical Series, vol. 28, $2^{\text {nd }}$ edn. Princeton University Press, Princeton, New Yersey (1972). First published in 1970

30. Rockafellar, R.T., Wets, R.J.B.: Variational analysis, Grundlehren der Mathematischen Wissenschaften [Fundamental Principles of Mathematical Sciences], vol. 317. Springer-Verlag, Berlin (1998) 